\title{
Experimental and Numerical Investigation of Flow Properties of Supersonic Helium-Air Jets
}

\author{
Steven A. E. Miller* \\ NASA Langley Research Center, Mail Stop 461, Hampton, VA 23681, U.S.A. \\ Jeremy Veltin ${ }^{\dagger}$ \\ The Pennsylvania State University, University Park, PA 16802, U.S.A.
}

\begin{abstract}
Heated high speed subsonic and supersonic jets operating on- or off-design are a source of noise that is not yet fully understood. Helium-air mixtures can be used in the correct ratio to simulate the total temperature ratio of heated air jets and hence have the potential to provide inexpensive and reliable flow and acoustic measurements. This study presents a combination of flow measurements of helium-air high speed jets and numerical simulations of similar helium-air mixture and heated air jets. Jets issuing from axisymmetric convergent and convergent-divergent nozzles are investigated, and the results show very strong similarity with heated air jet measurements found in the literature. This demonstrates the validity of simulating heated high speed jets with helium-air in the laboratory, together with the excellent agreement obtained in the presented data between the numerical predictions and the experiments. The very close match between the numerical and experimental data also validates the frozen chemistry model used in the numerical simulation.
\end{abstract}

\section{Nomenclature}

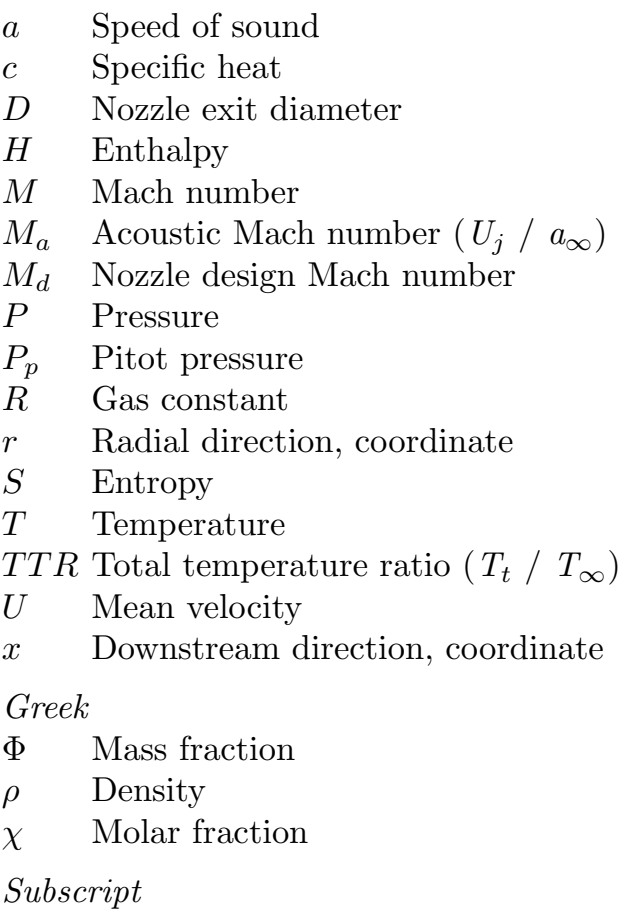

* Research Aerospace Engineer, Aeroacoustics Branch, AIAA Member.

${ }^{\dagger}$ Post-doctoral researcher, Aerospace Engineering Department. 
2 Quantity measured behind the shock created by the pitot probe

$j \quad$ Jet exit plane mean quantity

$t \quad$ Total or stagnation quantity

$\infty \quad$ Atmospheric quantity

Superscript

air Quantity relating to air

He Quantity relating to helium

heated Quantity relating to heated air

mix Quantity relating to a helium-air mixture

\section{Introduction}

Military aircraft jet engines have noise characteristics much louder than civilian aircraft due to their very low bypass ratio, high exhaust temperature, and high exhaust velocities. The high intensity noise generated by these heated subsonic or supersonic jets is a health hazard to ground crews as well as an annoyance to communities in the vicinity of airfields. This has led to a need for reduction of jet noise by developing noise suppression mechanisms that include new nozzle design concepts such as chevrons, corrugations, beveled nozzles, or other non-axisymmetric geometries. Research by Tanna et al. ${ }^{1}{ }^{\mathrm{L}} \mathrm{Lu},{ }^{2}$ Seiner et al. ${ }^{3}$ and Tam and $\mathrm{Chen}^{4}$ demonstrated that there are significant differences in how moderately heated supersonic jets generate noise compared with their unheated counterparts. Mixing noise is present in all jets and is caused by the coherent and incoherent turbulent structures. Adding some heating to a supersonic air jet helps to stabilize the instability waves in the shear layer, but when these instability waves travel faster than the ambient speed of sound, Mach wave radiation occurs in addition to the mixing noise. Jets operating supersonically and off-design also exhibit shock noise which is dominant in the upstream direction.

To study jets under realistic engine operating conditions, they should be heated so that the convection velocity of the turbulent structures is supersonic and the effects of Mach wave radiation can be measured. In practical terms, a heated jet facility involves significant complexity and expenses. To avoid this, the low density and high velocity of hot jets have been simulated in previous studies ${ }^{5-7}$ by using a lower density gas with different properties than air, namely, helium. By using a helium-air mixture, it becomes possible to simulate either the density or the acoustic velocity of a heated jet without having to heat the air. Although there are some differences between an actual heated jet and the above described simulated experiment, the major features of the noise generation process seem well represented and provided very satisfactory results in previous studies. ${ }^{5-7}$ However, as noise reduction concepts are being investigated, with increasing demand on the accuracy of the acoustic and flow measurements, a detailed assessment of the limits of the simulation of hot air jets with helium-air mixtures needs to be made. The present study investigates the details of the flow properties of both heated and heat simulated jets, using a combination of experimental measurements and Computational Fluid Dynamic (CFD) simulations. Experiments performed with cold, pure air jets and with helium-air mixtures highlight the effects of simulated heat on the jet flow properties. Numerical results with cold and heated pure air jets as well as helium-air mixture jets are used to assess the validity of the helium addition to properly simulate heat as well as validate a multi-species diffusion model.

\section{Approach}

The purpose of this study is to provide detailed comparisons between the flow properties of helium-air mixture jets simulating heated jets with heated jets. A short description of the simulation of heated jets with a helium-air mixture is presented. Then the experimental and computational approaches are described.

\section{A. Simulating Heating with Helium-Air Mixtures}

In order to make acoustic measurements that can be directly compared to aircraft engine measurements, the temperature of the jet is an important parameter that needs to be replicated. A heated jet has different physical characteristics than a cold jet, due to the increase in jet exit velocity and decrease in jet density. This affects the acoustical properties of the jet. In order to avoid the power, infrastructure, and operating 
cost requirements of a heated jet facility, a helium-air mixture can be used as a means to simulate the desired jet properties without actually heating the air flow. ${ }^{8}$ This methodology was developed at the Pennsylvania State University by Kinzie and McLaughlin ${ }^{6}$ and Doty and McLaughlin. ${ }^{5}$ A similar methodology is being used by Papamoschou ${ }^{7}$ at the University of California Irvine. The quantities that need to be matched when doing acoustic measurements in hot jets are the jet acoustic velocity $a_{j}$ and the jet density $\rho_{j}$. Helium was chosen for its low density and high acoustic velocity and the fact that it is an inert gas which is inexpensive to obtain and handle. In order to account for varying atmospheric conditions, the characterizing parameter is the jet exit to ambient temperature ratio $T_{j} / T_{a}$. For the helium-air jet to match a corresponding heated jet, two non-dimensional groups are required: the density ratio $\rho_{j} / \rho_{a}$ and the acoustic velocity ratio $a_{j} / a_{a}$. It is possible to match both, as mentioned by Kinzie, ${ }^{6}$ by mixing some helium with ambient air. However, it is impractical in a large facility. In prior experimental work by Doty and McLaughlin, ${ }^{5}$ the two parameter matching methods were investigated, with direct comparisons to heated air measurements. Only very small variations in the acoustic spectra were observed and were of the same order of magnitude as the experimental uncertainty of the measurements. More recent careful comparisons ${ }^{9}$ with measurements performed in other facilities have shown very good agreement when matching the acoustic velocity of the mixture jet to that of a heated jet. Therefore, matching of the acoustic velocity is chosen for the experimental and numerical work presented in this study, meaning the mixture properties are defined such that:

$$
\left(\frac{a_{j}}{a_{a}}\right)^{\text {heated }}=\left(\frac{a_{j}}{a_{a}}\right)^{\text {mix }} \Leftrightarrow \sqrt{\gamma R T_{j}}=\sqrt{\gamma^{\text {mix } R^{\text {mix }} T_{j}^{\text {mix }}}}
$$

More details on the effectiveness of helium in simulating hot jets, as well as the implications associated with this methodology are discussed in Doty and McLaughlin ${ }^{5}$ and Papamoschou. ${ }^{7}$ These two references discuss the safety and economic benefits of helium-air mixtures compared to heating air for the experiments conducted in this facility.

\section{B. Experimental Approach}

Experimental measurements of the mean flow properties were performed using Pitot probes within the jet plume. The schematic diagram presented in Fig. 1a shows the nomenclature used for the flow quantities around a single probe. A subscript ( $)_{2}$ indicates the properties just downstream of the normal shock portion of the shock wave, immediately upstream of the Pitot probe, and the subscript ( $)_{1}$ indicates the local properties ahead of the shock. Figure $1 \mathrm{~b}$ and $1 \mathrm{c}$ show photographs of the Pitot probes used for the measurements. It consists of a rake of five probes of outer diameter $0.6 \mathrm{~mm}(0.025 ")$ and inner diameter $0.25 \mathrm{~mm}(0.01 ")$. Apart from this new probe rake, the overall experimental setup is the same as the one described in Miller et al. ${ }^{10}$
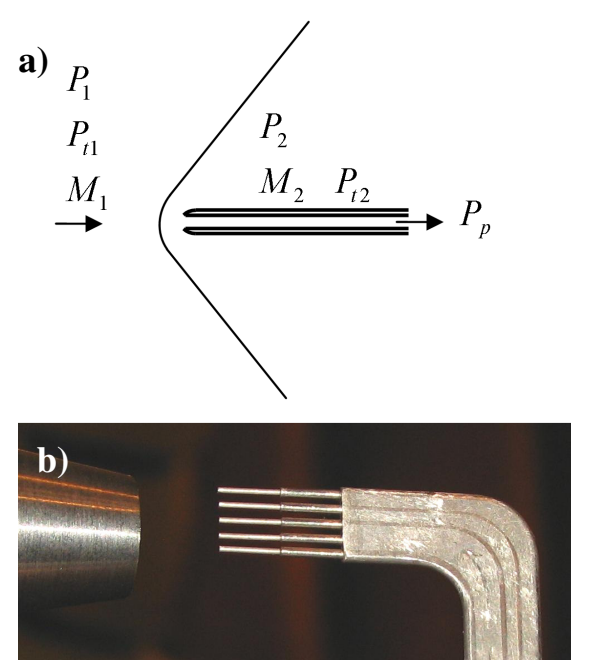

Figure 1. a) Schematic diagram of a Pitot pressure probe in a free stream flow, b) detailed view of the Pitot rake at the exit plane of a round nozzle, c) Pitot setup.

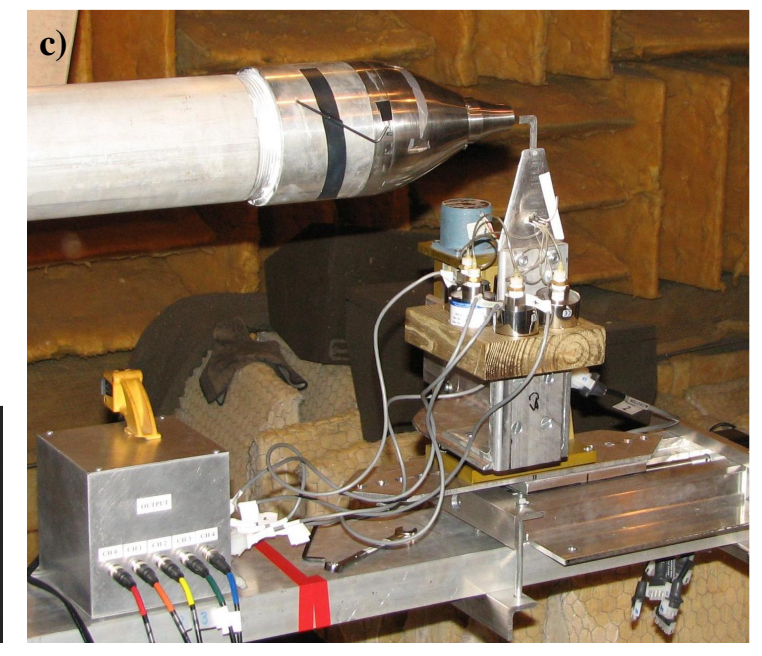


The Pitot pressure $P_{p}$, can be used to find a value for the local Mach number $M_{1}$ using a similar process developed for pure air jets. Wherever the flow is supersonic, a shock forms in front of the probe, and $P_{p}$ is equal to the total pressure behind the shock, $P_{t 2}$. $M_{1}$ is then calculated using the Rayleigh Pitot formula shown in Eq. (2) written with static pressure before the shock. Wherever the flow is subsonic, there is no shock ahead of the probe, thus $P_{p}$ is equal to $P_{t 1}$ and the isentropic flow formula of Eq. (3) can be used. The jets evaluated in this study are fully-expanded, the static pressure $P_{1}$ can be assumed to be constant and equal to the ambient static pressure $P_{\infty}$ everywhere within the jet. A more detailed description of this processing is presented in Miller et al. ${ }^{10}$ as well as some detailed comparisons between simulations and experimental measurements.

$$
\begin{gathered}
\frac{P_{p}}{P_{1}}=\left[\frac{(\gamma+1) M_{1}^{2}}{(\gamma-1) M_{1}^{2}+2}\right]^{\frac{\gamma}{\gamma-1}}\left[\frac{\gamma+1}{2 \gamma M_{1}^{2}-(\gamma-1)}\right]^{\frac{1}{\gamma-1}} \\
\frac{P_{p}}{P_{1}}=\left[1+\frac{(\gamma-1)}{2} M_{1}^{2}\right]^{\frac{\gamma}{\gamma-1}}
\end{gathered}
$$

However, attempting to perform this calculation in a helium-air mixture jet leads to a new complication: the mass concentration of helium $\Phi_{1}^{H e}$ is not constant within the mixing layer, leading to fluctuations of properties such as the local specific heat ratio $\gamma_{1}$ across the jet. Since no measurement of the species concentration is available within such high speed jets, an analytical approach needs to be used in order to yield estimates of the gas constitution. In steady axisymmetric turbulent jets the conservation of momentum in the $x$ (streamwise) direction can be expressed as follow:

$$
\rho u \frac{\partial u}{\partial x}+\rho v \frac{\partial u}{\partial r}-\frac{\partial}{\partial r}\left(\mu_{t} \frac{\partial u}{\partial r}\right)=-\frac{\partial P}{\partial x}
$$

where $\mu_{t}$ represents the eddy viscosity, or the effective viscosity of a turbulent fluid, and $u$ and $v$ are respectively the axial and radial components of the velocity. Note that the normal stress contribution is neglected. In the free-stream jets considered, the streamwise gradient of pressure can be neglected $(\partial P / \partial x)$, such that the right hand side term is dropped. Then, within the jet mixing layer, three different phenomena can cause mixing of the jet inner gas mixture with the ambient air: turbulent mixing, molecular diffusion, and thermal diffusivity. Turbulent mixing can be described, as shown by Spalding, ${ }^{11}$ using the conservation of mass of the entrained flow. The resulting equation relates the mass concentration of helium $\Phi^{H e}$ to the spatial velocity field:

$$
\rho u \frac{\partial \Phi^{H e}}{\partial x}+\rho v \frac{\partial \Phi^{H e}}{\partial r}=\frac{\partial}{\partial r}\left(\frac{\mu_{t}}{S c_{t}} \frac{\partial \Phi^{H e}}{\partial r}\right)
$$

where $S c_{t}$ refers to the turbulence Schmidt number. When mixing occurs via molecular and thermal diffusion (of helium), rather than turbulent mixing, then the following equation, based on Fick's law and derived by Schlichting ${ }^{12}$ is used:

$$
\rho u \frac{\partial \Phi^{H e}}{\partial x}+\rho v \frac{\partial \Phi^{H e}}{\partial r}=\frac{\partial}{\partial r}\left[\rho D_{12}\left(\frac{\partial \Phi^{H e}}{\partial r}+k_{T} \frac{\partial(\ln T)}{\partial r}\right)\right]
$$

where $D_{12}$ denotes the coefficient of binary diffusion of helium in air and $k_{T}$ the thermal diffusion ratio. However, even though the jets of interest are not isothermal, they are unheated and therefore do not have large enough temperature gradients to produce appreciable thermal diffusion relative to other mechanisms. The thermal diffusion term can therefore be neglected. The remaining molecular diffusivity term is compared with the right hand side term of Eq. (5). The coefficient of molecular diffusion of helium in air is a known quantity, with value $D_{12} \simeq 62.10^{-6} \mathrm{~m}^{2} / \mathrm{s}$ at ambient conditions. Therefore, the term $\rho D_{12}$ is of the order of magnitude $10^{-4}$. Also, the turbulence kinematic viscosity $\nu_{t}$ can be estimated, as shown by Schlichting ${ }^{12}$ and by Spalding ${ }^{11}$ as $\nu_{t}=0.026 \times r_{0.5} \times U_{c}$, where $r_{0.5}$ is the half velocity point and $U_{c}$ is the centerline velocity of the jet. This leads to a dynamic viscosity $\mu_{t}$ with an order of magnitude $10^{-1}$ for the jets considered $\left(D=0.0127 \mathrm{~m}\right.$ and $U_{j}=300$ to $\left.600 \mathrm{~m} / \mathrm{s}\right)$. Using an estimated value of 0.7 for the turbulent Schmidt number, $S c_{t}$, as suggested by Panchapakesan and Lumley, ${ }^{13}$ the constant in the right hand side of Eq. (5) has an order of magnitude of $10^{-1}$. This is much higher than the order of magnitude $10^{-4}$ obtained for the molecular diffusion. Therefore, molecular diffusion is neglected, which leaves turbulent mixing as the sole 
contributor to the diffusion of helium outside of the jet. Eq. (4) and Eq. (5) are therefore the only equations to consider. Previous studies ${ }^{13,14}$ have shown that while the value of $S c_{t}$ varies across the shear layer, a constant value of 0.7 can be used and produces adequate concentration estimates. Therefore, $S c_{t}$ and $\mu_{t}$ are assumed constant and moved outside the partial derivatives. Furthermore, assuming the mass concentration of helium can be expressed as a sole function of the local flow velocity $U_{1}$, the chain rule for partial derivative can be applied to Eq. (5), and then combined with Eq. (4). These mathematical operations yield:

$$
\left(\frac{1}{S c_{t}}-1\right) \frac{\partial \Phi^{H e}}{\partial U_{1}} \frac{\partial^{2} U_{1}}{\partial r^{2}}+\frac{\partial^{2} \Phi^{H e}}{\partial U_{1}^{2}}\left(\frac{\partial U_{1}}{\partial r}\right)^{2}=0
$$

In order to obtain a solution for this second order differential equation the function $U_{1}(r)$ must be known. It was observed in past experimental studies ${ }^{15}$ that the velocity profile in a jet follows a Görtler error function distribution, with amplitude proportional to the jet velocity $U_{j}$. The first and second derivative of such a function is also proportional to $U_{j}$. As a result, the second term of Eq. (7) is proportional to the square of the convection velocity, which means the first term can be neglected. Eq. (7) then reduces to a simple linear variation of the mass concentration of helium with respect to velocity. Following this development, reduction of the pressure data is performed with the underlying assumption that the helium concentration varies linearly with local velocity. Numerical and experimental results shown in this paper will illustrate the validity of this argument.

The processing of the experimental data is performed with Matlab. The pitot pressure $P_{p}$ is first used for (using the Rayleigh and isentropic formula in the appropriate parts of the jet) an estimated value of the local Mach number $M_{1}$ by using $\gamma_{j}$ and $T_{j}$ everywhere within the jet. Then, a first estimate of the local velocity is obtained, as well as the local static temperature, $T_{1}$ using the Crocco relation. A better estimate of the velocity is then computed using $T_{1}$. Then the mass and molar concentration of the species are evaluated, leading to an estimate for the local specific heat ratio and gas constant of the mixture. A refined velocity is computed from these values and the whole process is repeated until it converges to the final values for the local velocity $U_{1}$ and temperature $T_{1}$. Any other local property of the flow can be computed from these. The overall processing methodology is summarized in the flow chart of Fig. 2.

\section{Computational Approach}

In the previous section the experimental method and approach to find field-variables from helum-air jets is described. A numerical approach to find the same field-variables at the same spatial locations is discussed here. This numerical approach uses the Reynolds Averaged Navier-Stokes (RANS) equations, which are numerically integrated until a steady solution is achieved with the NPARC Alliance Wind-US 2.0 solver. Wind-US was developed by the NPARC Alliance which is a parternship between NASA Glenn Research Center and the U. S. Air Force Arnold Engineering Development Center, and additional contributors. A large number of validation cases of a broad range of flow-regimes have been made with the Wind-US solver by the NPARC Alliance. Wind-US can solve the Euler or Navier-Stokes equations in conjunction with many different turbulence models on structured or unstructured multi-block domains. It also has the ability to perform the computations in serial or parallel using the Message Passing Interface or the Parallel Virtual Machine. The Menter ${ }^{16}$ Shear Stress Transport (SST) turbulence model is used in the present simulations for both heated air and helium-air mixtures to close the steady RANS equations. In addition, transport equations are solved for each species of the flow. In this case a helium, oxygen, and nitrogen mixture is chosen to represent the helium-air mixture. The traditional ideal gas model is also used for corresponding heated air simulations with a constant $\gamma$. The turbulent Prandtl number is set in all simulations at 0.90 and $S c_{t}$ is set as 0.70 .

Generally CFD simulations of nozzles use ideal gas such as air because corresponding laboratory measurements use unheated or heated air. CFD simulations assume that the gas constant and ratio of specific heats are constant. Unfortunately, this assumption does not allow for the variation of the thermodynamic quantities inside and outside the nozzle, where they differ because of the large temperature differences. In aircraft jet engines the combustion process adds additional species to air changing the thermodynamic quantities. Simulation of these combustion reactions is computationally very expensive. However, in conventional aircraft engines the majority of the combustion process is complete when the gas reaches the nozzle inlet. Therefore, a frozen chemistry model can be used. The assumption can also be made, as discussed in the previous section, that diffusion is the only mechanism that changes the species concentration. Similar as- 


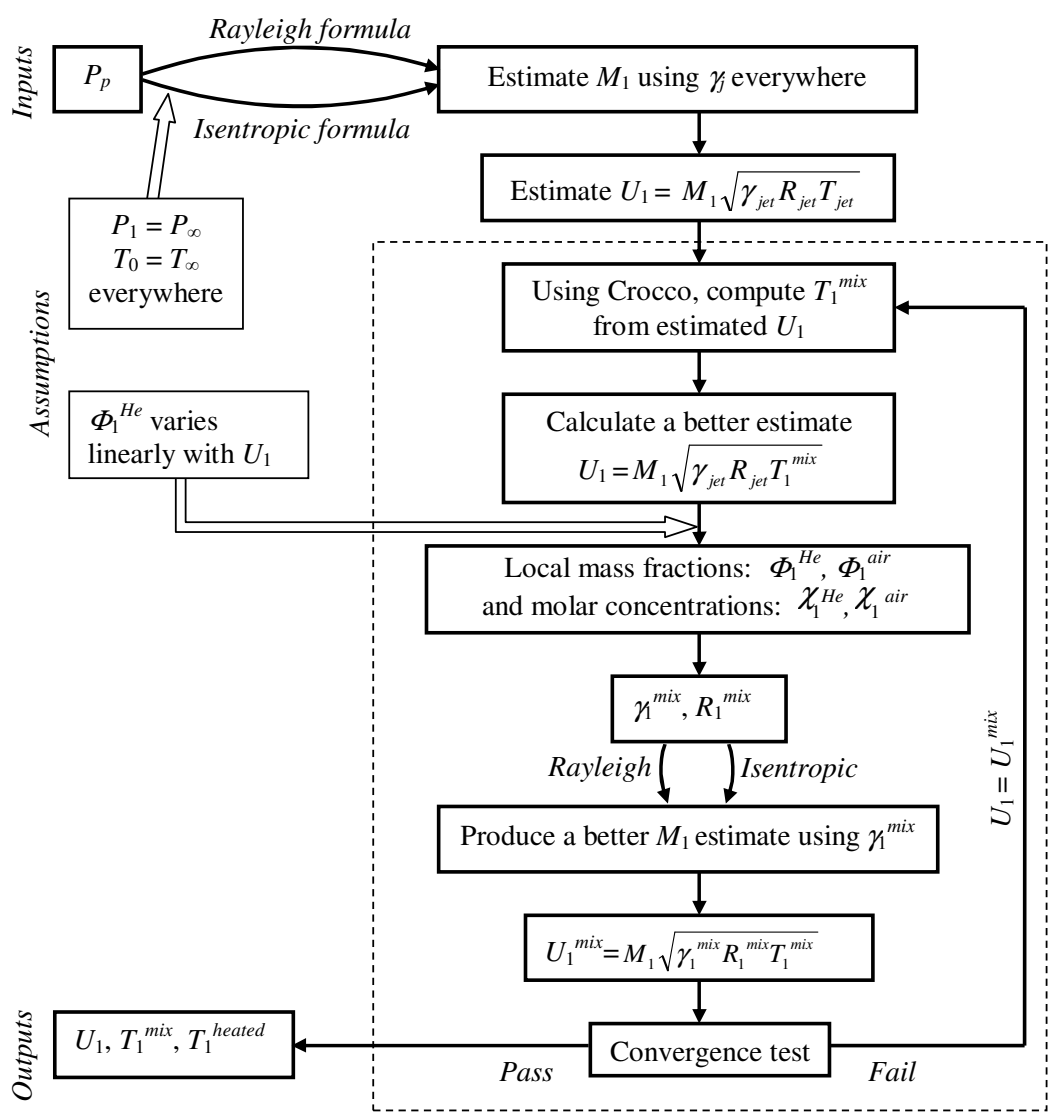

Figure 2. Flowchart of the pitot measurement processing.

sumptions were made by Yoder et al. ${ }^{17}$ with comparison between the effects of using a ideal gas (air), ideal gas mixtures, and a frozen exhaust gas mixture of subsonic and supersonic jets.

In a helium-air mixture jet there is no combustion process and the number of species are relatively small compared to those produced in an aircraft engine. Since the helium-air mixture is known in the experiments and the only mixing occurs upstream of the nozzle, the mass species concentration is well known at the nozzle outlet. Far outside of the jet, the gas mixture corresponds to ambient air. Since no chemical process occurs in the shear layer of the jet, and mixing is mainly produced by turbulence, use of frozen chemistry in Wind-US is used. Frozen chemistry essentially assumes that the reaction source terms are zero and in these simulations only the diffusion and turbulent mixing of individual species is modeled.

Wind-US has the ability to perform reacting flow calculations with a preexisting chemistry model and is initialized with the addition of a keyword in the Wind-US input file. Frozen chemistry modeling was used for the helium-air simulations presented in this study, but not for the heated air simulations. Additional inputs required by the chemistry module consist of the species concentration in the far-field and at the inflow boundary at the nozzle inlet. The full implementation of the frozen chemistry module and associated transport equations without reaction sources can be found in the Wind-US Documentation ${ }^{18}$ or the Wind-US Developer's Reference.

Wind-US has a number of standard chemistry packages that contain various models of standard molecules. Unfortunately, none of these packages contain information for the element helium. An external chemistry file is developed by Joo Kyung Suh of the Raytheon Corporation that contains the species helium along with the two most abundant elements in air, oxygen and nitrogen. The chemistry file contains the thermodynamic coefficients of the three species, derived from McBride et al. ${ }^{19}$ where three curves are defined for each species. Each of these species has a set of three curves that describe the specific heat at constant pressure, enthalpy, and entropy over a given range of temperatures in terms of coefficients $a_{i}$ and $b_{i}$. Ranges of temperature for 
the three curves are from $200 \mathrm{~K}$ to $20,000 \mathrm{~K}$. Thermodynamic values defined by McBride et al. ${ }^{19}$ based on the coefficients in the helium-air chemistry file are,

$$
\begin{gathered}
c_{p}^{0} / R=a_{1} T^{-2}+a_{2} T^{-1}+a_{3}+a_{4} T+a_{5} T^{2}+a_{6} T^{3}+a_{7} T^{4}+a_{8} T^{5} \\
H^{0} /(R T)=-a_{1} T^{-2}+a_{2} T^{-1} \ln T+a_{3}+a_{4} T / 2+a_{5} T^{2} / 3+a_{6} T^{3} / 4+a_{7} T^{4} / 5+a_{8} T^{5} / 6+b_{1} / T \\
S^{0} / R=b_{2}-a_{1} T^{-2} / 2-a_{2} T^{-1}+a_{3} \ln T+a_{4} T+a_{5} T^{2} / 2+a_{6} T^{3} / 3+a_{7} T^{4} / 4+a_{8} T^{5} / 5
\end{gathered}
$$

where $c_{p}$ is the specific heat at constant pressure, $H$ is the enthalpy, $R$ is the universal gas constant, $S$ is the entropy, and $T$ is the temperature. The chemistry file also contains the reaction rate coefficients for the three species with which Wind-US calculates the forward and backward reaction rates. Both rates are independent and are of the form,

$$
k=C T^{S} \exp \left[-D / K_{b} T\right]
$$

where $K_{b}$ is the Boltzmann constant, $k$ is either the forward or backward reaction rate, and $C$ and $D$ are reaction rate coefficients. Since only a three species model is available, other species that are typically present in small quantity in air, such as Argon, are ignored. A similar assumption was made successfully by Yoder et $a l .{ }^{17}$ The frozen chemistry model of a helium-air mixture has not been tested on high speed subsonic or supersonic on- or off-design jets before. These simulations represent the first steady helium-air mixture jet simulations for this flow regime.

To match experiments, axisymmetric simulations are conducted, using computational grids developed and described in more details in a previous study ${ }^{10}$ The axisymmetric form of the governing equations are solved. Nozzle coordinates are consistent with those used in experiments. Two computational grids are used for this study. The first is for the converging nozzle, $M_{d}=1.0$ and the second is for the converging-diverging nozzle, $M_{d}=1.5$. The computational domain extends $75 D$ from the nozzle exit in the downstream direction, $50 \mathrm{D}$ from the nozzle exit in the radial direction, and $5 \mathrm{D}$ upstream from the nozzle inlet. Initial iterations are performed using a constant CFL number of 0.50 and then explicit Euler time stepping using a constant time step is used until the $L^{2}$ residual remains constant and a visual inspection of the solution appears steady. The $L^{2}$ residual is a global measure of the residual error of the governing equations of the flow-field. Grid independence studies are performed by using every other grid point in the computational domain in both the streamwise and radial directions. No difference in the steady solution between the sequenced (every other grid point used) and unsequenced grid is apparent in any of the solutions. The boundary layer at the nozzle exit is fully resolved by setting the first grid point in the viscous sublayer region using $y^{+}=1$, where $y^{+}$is the coordinate of the viscous wall layer using average conditions. ${ }^{12}$

Boundary conditions for the simulations consist of a downstream outflow in the streamwise direction allowing the flow to exit the computational domain. This assumes that the outflow is subsonic and the freefield pressure is set as the ambient pressure. Free-stream boundary conditions are specified in the free-field where a small uniform flow at $M_{\infty}=0.001$ is assumed for stability and the total ambient pressure is equal to the downstream static pressure. A standard axisymmetric boundary condition, which is essentially an inviscid wall, lies on the centerline at $y / D=0$. Heat transfer is not considered in the study and adiabatic no-slip wall boundary conditions are used on the nozzle surface. Finally, the upstream boundary condition for the nozzle inlet specifies the total pressure and total temperature of the jet with an initial inlet $M=0.15$ normal to the inlet plane. The inlet $M$ may fluctuate and the total values of $P$ and $T$ are held constant. Full details of the computational grids and boundary conditions used in this simulation can be found in Miller et al. ${ }^{10}$ The numerical implementation and mathematical development are shown in Nelson and Power. ${ }^{20}$ These boundary conditions are sufficient for a steady solution to be found when using an ideal gas. Some of the simulations use a helium-air mixture, and thus the mass concentration of the helium species is needed at the free-stream and inlet boundary conditions. In the free-stream the mass concentration of helium is zero and at the nozzle inlet the mass concentration is set to match $M_{a}$ at the nozzle exit. When helium-air simulations are performed $T T R=1.0$ at the nozzle inlet.

\section{Summary of Jet Operating Conditions}

Experimental measurements were performed in the Pennsylvania State University jet noise facility with pure air and helium-air mixture jets. No experimental measurements were performed with heated air jets as the facility does not have this capability. CFD simulations were performed using Wind-US for both heated jets 
and the corresponding helium-air mixtures. The jet conditions experimentally and numerically investigated are summarized in Table 1.

Two nozzles are used for the experimental measurements: a contoured, purely converging (design Mach number $M_{d}=1.0$ ) nozzle and a converging-diverging nozzle designed with the method of characteristics to operate shock-free at a Mach number $M_{d}=1.5$. The coordinates of these nozzles are imported directly into Gridgen software to create the computational domains for the corresponding Wind-US simulations.

Table 1. Jet operating conditions for the numerical simulations and experiments.

\begin{tabular}{ccc|ccc|ccccc}
$M_{d}$ & $M_{j}$ & TTR & $\begin{array}{c}U_{j} \text { heated } \\
(\mathrm{m} / \mathrm{s})\end{array}$ & $M_{a}$ & $\begin{array}{c}T_{j} \\
(\mathrm{~K})\end{array}$ & $\begin{array}{c}\chi_{j}{ }^{H e} \\
(\%)\end{array}$ & $\begin{array}{c}\Phi_{j}{ }^{H e} \\
(\%)\end{array}$ & $\gamma_{j}{ }^{\text {mix }}$ & $\begin{array}{c}R_{j}{ }^{\text {mix }} \\
\left(\mathrm{m}^{2} \mathrm{~K} / \mathrm{s}^{2}\right)\end{array}$ & $\begin{array}{c}a_{j}{ }^{\text {mix }} \\
(\mathrm{m} / \mathrm{s})\end{array}$ \\
\hline 1.0 & 0.9 & 1.0 & 286 & 0.84 & 252 & 0 & 0 & 1.4 & 287 & 307 \\
1.0 & 0.9 & 2.22 & 427 & 1.26 & 240 & 61.4 & 18.0 & 1.53 & 609 & 473 \\
1.0 & 0.9 & 3.6 & 543 & 1.60 & 236 & 81.7 & 38.1 & 1.59 & 969 & 604 \\
1.0 & 1.47 & 2.2 & 626 & 1.84 & 186 & 63.5 & 19.4 & 1.54 & 635 & 426 \\
1.5 & 1.5 & 1.0 & 427 & 1.26 & 203 & 0 & 0 & 1.4 & 287 & 285 \\
1.5 & 1.5 & 1.62 & 544 & 1.60 & 190 & 44.7 & 10.1 & 1.49 & 467 & 363 \\
1.5 & 1.5 & 2.22 & 637 & 1.87 & 184 & 63.7 & 19.5 & 1.54 & 636 & 423 \\
1.5 & 1.5 & 3.6 & 811 & 2.39 & 175 & 84.1 & 42.3 & 1.60 & 1044 & 542
\end{tabular}

\section{Results}

This section presents experimental and numerical results from the jet conditions summarized in Table 1. The streamwise velocities at two traverse locations, $x / D=4$ and $x / D=8$, for both the $M_{j}=0.9$ and $M_{j}=1.5$ jets at all temperature ratios are shown first, in Figs. 3 and 4 . The numerical predictions at $T T R=1$ are obtained with pure air, while the predictions of the $T T R=2.2$ and $T T R=3.6$ cases are obtained with both heated air and helium air-mixtures. Experimental results are plotted as points, numerical results with heated air as solid lines, and numerical results with helium-air mixtures as dashed line. The first observation that can be made is that the numerical results from both methods collapse extremely well. This illustrates that there is no difference between the predicted velocity profile of a heated air jet and the corresponding helium-air jet. This demonstrates that the helium-air mixture provides velocity profiles that are similar to the corresponding heated jet condition. The experimentally determined velocity profiles also show good agreement with the numerical predictions. However, small discrepancies appear in the inner part of the mixing layer, where the experiments generally show lower velocity than the numerical results. CFD results of high speed subsonic jets demonstrate lower mixing close to the nozzle exit relative to experimental data. In supersonic jets near $M_{j}=1.5$, compressibility effects lower the mixing rate in the experiment, that in turn agree better with CFD results. These discrepancies are more visible in the non-dimensionalized plots presented later. The centerline velocity obtained from both the CFD and the Pitot measurements increases with increasing (simulated) TTR for a jet of given $M$. These velocities match the theoretical values presented in Table 1. In the region of the outer part of the jet, $r / D \geq 0.6$, the effects of helium (or heat) addition on the velocity profile is much less dramatic: the increase in velocity is very slight, and the curves quickly merge into one velocity profile. This is in agreement with measurements performed by Lau ${ }^{2}$ with laser velocimetry in $M_{j}=0.5$ heated jets of different temperature ratios. 

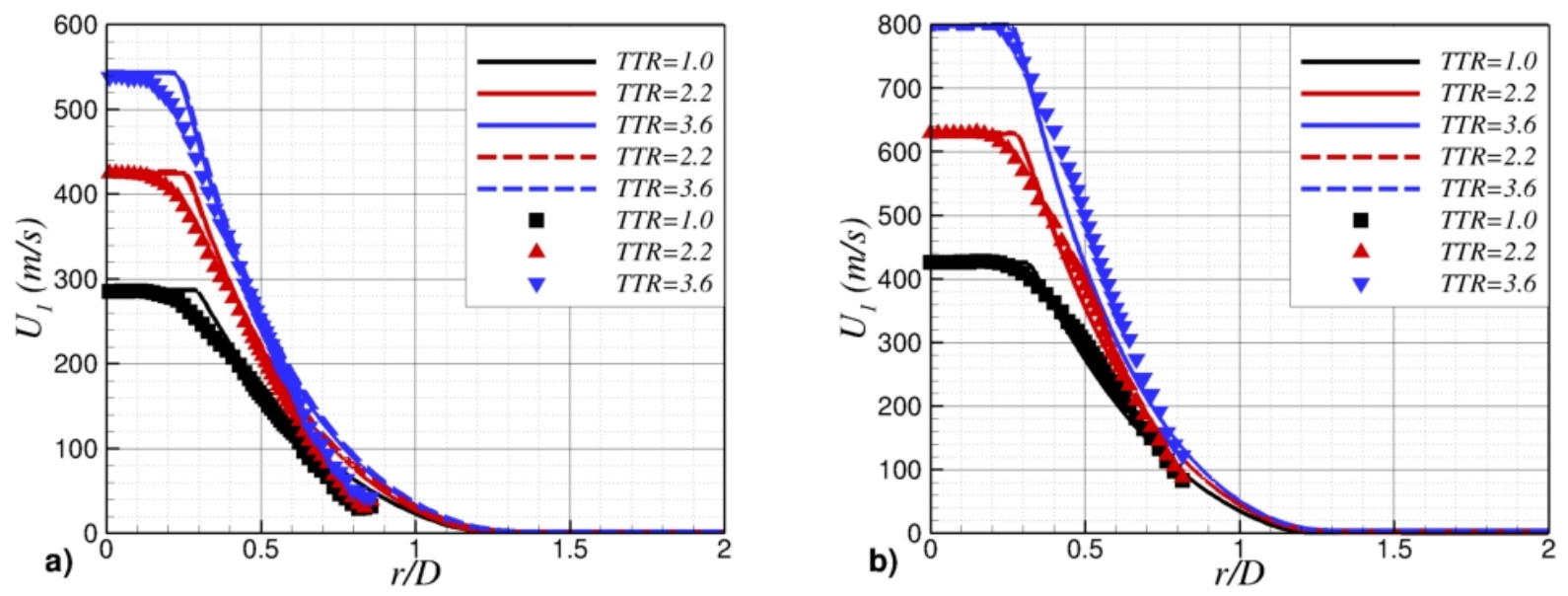

Figure 3. Velocity profiles measured in jets of various $T T R$ at $x / D=4$. Heated simulations, helium-air simulations, and experiments are represented by solid lines, dashed lines, and symbols respectively. a) $M_{j}=0.90$ b) $M_{j}=1.50$.
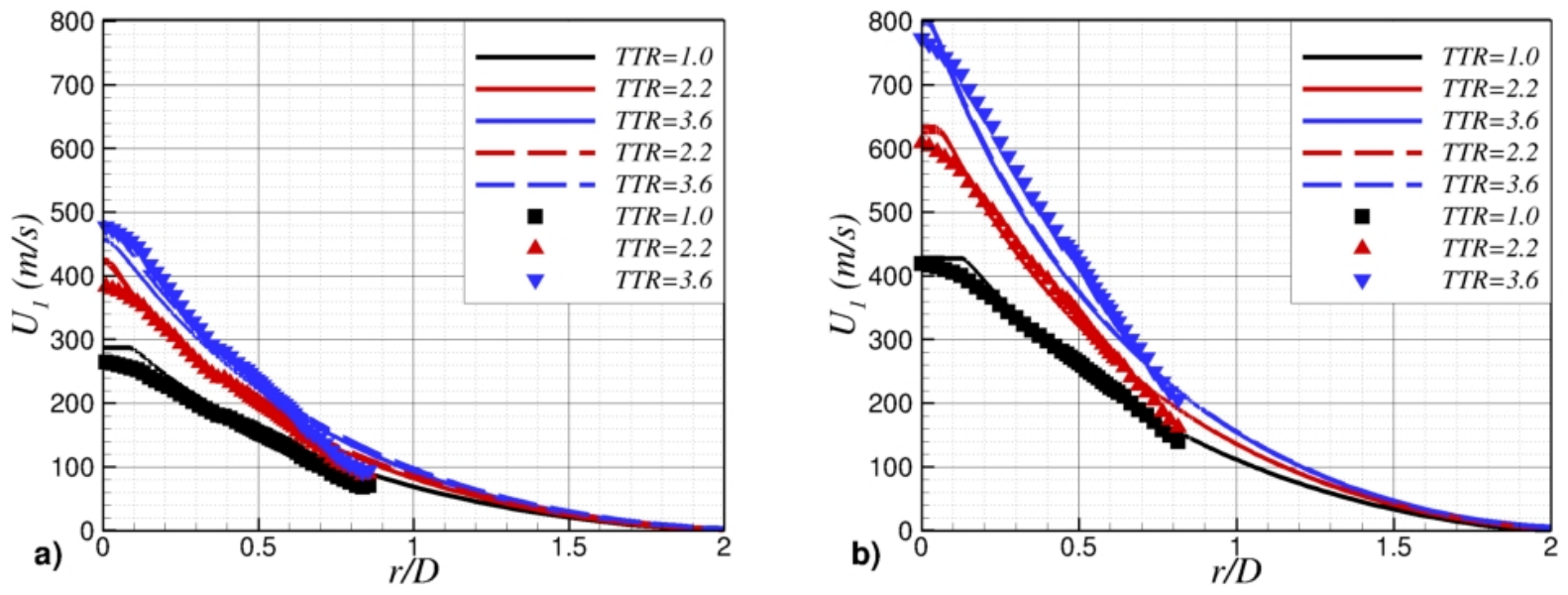

Figure 4. Velocity profiles measured in jets of various $T T R$ at $x / D=8$. Heated simulations, helium-air simulations, and experiments are represented by solid lines, dashed lines, and symbols respectively. a) $M_{j}=0.90$ b) $M_{j}=1.50$. 
It was also found by Lau et al. ${ }^{15}$ that the non-dimensionalized velocity profiles for isothermal jets could be perfectly collapsed when plotted as a function of the normalized radial distance $\eta=\left(r-r_{0.5} / \delta_{\omega}\right)$, where $\delta_{\omega}=U_{\max } /[d U / d r]_{\max }$ represents the vorticity thickness and $r_{0.5}$ is the half velocity point. The collapse was shown to follow a Görtler error function profile:

$$
U_{1} / U_{j}=\frac{1}{2}[1-\operatorname{erf}(\sigma \eta)]
$$

where $\sigma$ is an experimentally determined constant. Similar measurements by $\mathrm{Lau}^{2}$ showed the same collapse for heated jets. Performing a similar non-dimensionalization of both the experimental data and numerical predictions of this study, yields a very good collapse of the data, as shown in Figs. 5 and 6 . Both the CFD and the experimental results collapse very well across all temperature ranges and are therefore self-consistent. The helium-air simulation results also match very closely to the heated air results, providing confidence in the chemical frozen module of Wind-US. However, this representation highlights the discrepancies between the computational results and the experiments. The experimental results fit closely to an error function with coefficient $\sigma=-2.055$ for the $M_{j}=0.9$ jets and $\sigma=-2.073$ for the $M_{j}=1.5$ jets. On the other hand, the numerical results are over-predicting the velocity around $\eta= \pm 0.5$. This can be attributed to the limitation of the Menter SST model to correctly predict the spreading the jet correctly, as previously discussed. ${ }^{10}$

Since the velocity data obtained from experiment can be modeled by an error function, the first and second derivative of the velocity profile with respect to radial distance can easily be computed. In the previous section, Eq. (7) was solved by making the assumption that the second derivative of the velocity profile is negligible compared to the square of its first derivative. The two quantities can now be compared by plotting their ratio, as shown in Fig. 7. It is obvious from this plot that for any value $\eta$ comprised between -0.7 and 0.7 the ratio above mentioned is greater than 10 . Since this $\eta$ range corresponds to the whole mixing layer, this proves that the first term of Eq. (7) can indeed be neglected. The assumption originally made that the concentration of helium varies linearly with the streamwise velocity is valid.

Since Wind-US has the ability to calculate the mass concentration of the helium species of the frozen chemistry model spatially, a direct comparison can be conducted with the experiments to further validate the assumptions made. The helium mass concentration values are plotted for both experimental and numerical data against $u \mathrm{~m} / \mathrm{s}$ in Fig. 8 at radial locations $r / D=4$ and $r / D=8$. The experimental measurements are shown as scatter points and the computation is represented by lines. Clearly, each set of either numerical or experimental data shows an almost perfect linear relationship between the velocity and helium mass concentration. For the supersonic on- and off-design jets the agreement between the slope of the prediction and slope of the experiment is almost perfect. The predictions for TTR $=2.2$ and $T T R=3.6$ of all three $M_{j}$, are extremely similar and in the figure can not be differentiated easily. The experimental data agrees in a similar fashion except for the $M_{j}=0.9$ cases which show some differences between the $x / D=4$ and $x / D=8$ locations. This is attributed to the fact that the measurements are downstream of the potential core, which results in a lower centerline velocity for the experimental data. The predicted potential core length does not perfectly match, due to the under-prediction of the spreading rate discussed earlier. As a result, the larger centerline velocity creates the slight discrepancy in the slopes observed in Fig. 8. This (small) discrepancy is therefore not due to the helium-air frozen chemistry model, which is still believed to perform adequately. 


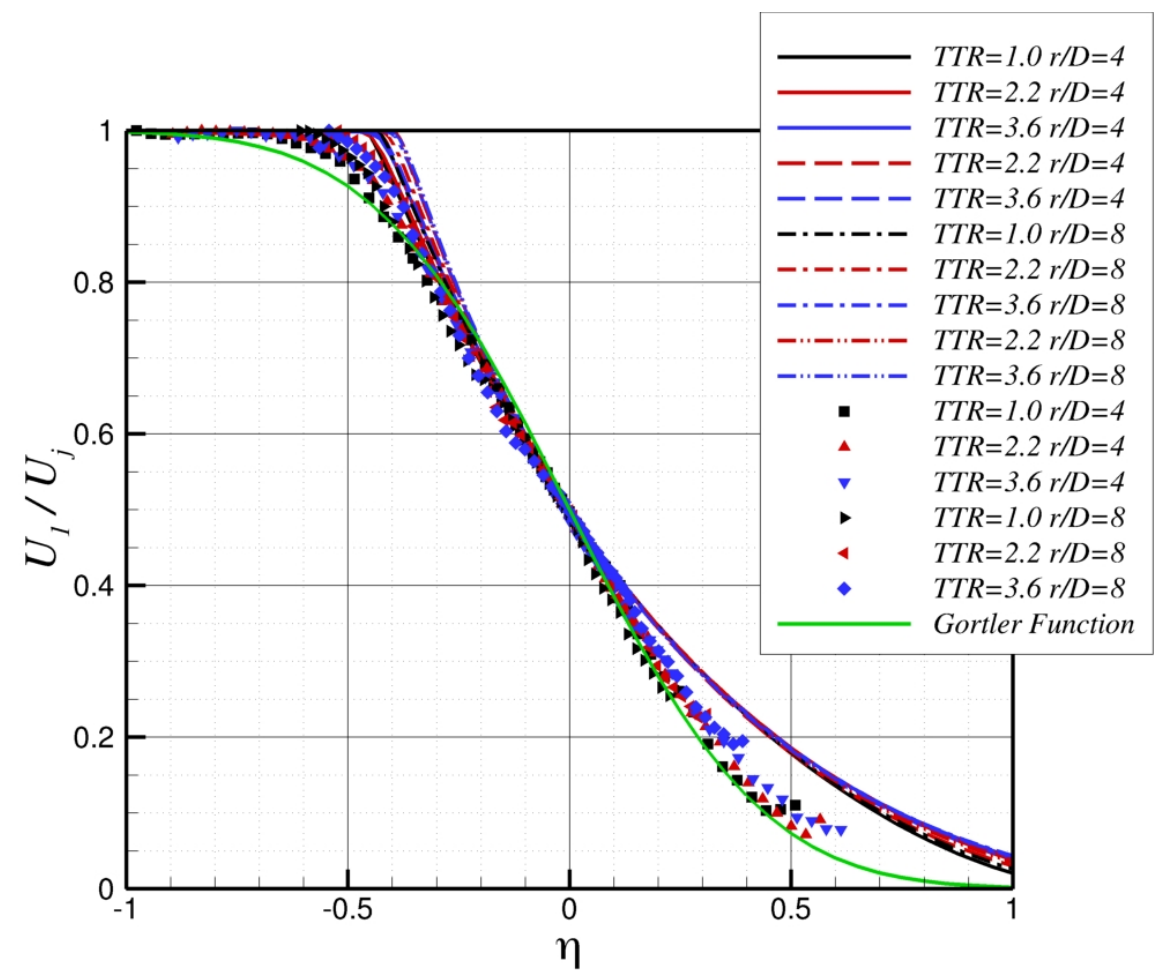

Figure 5. Non-dimensionalized radial mean velocity profiles measured in fully expanded $M_{j}=0.9$ jets of different simulated temperature ratios.

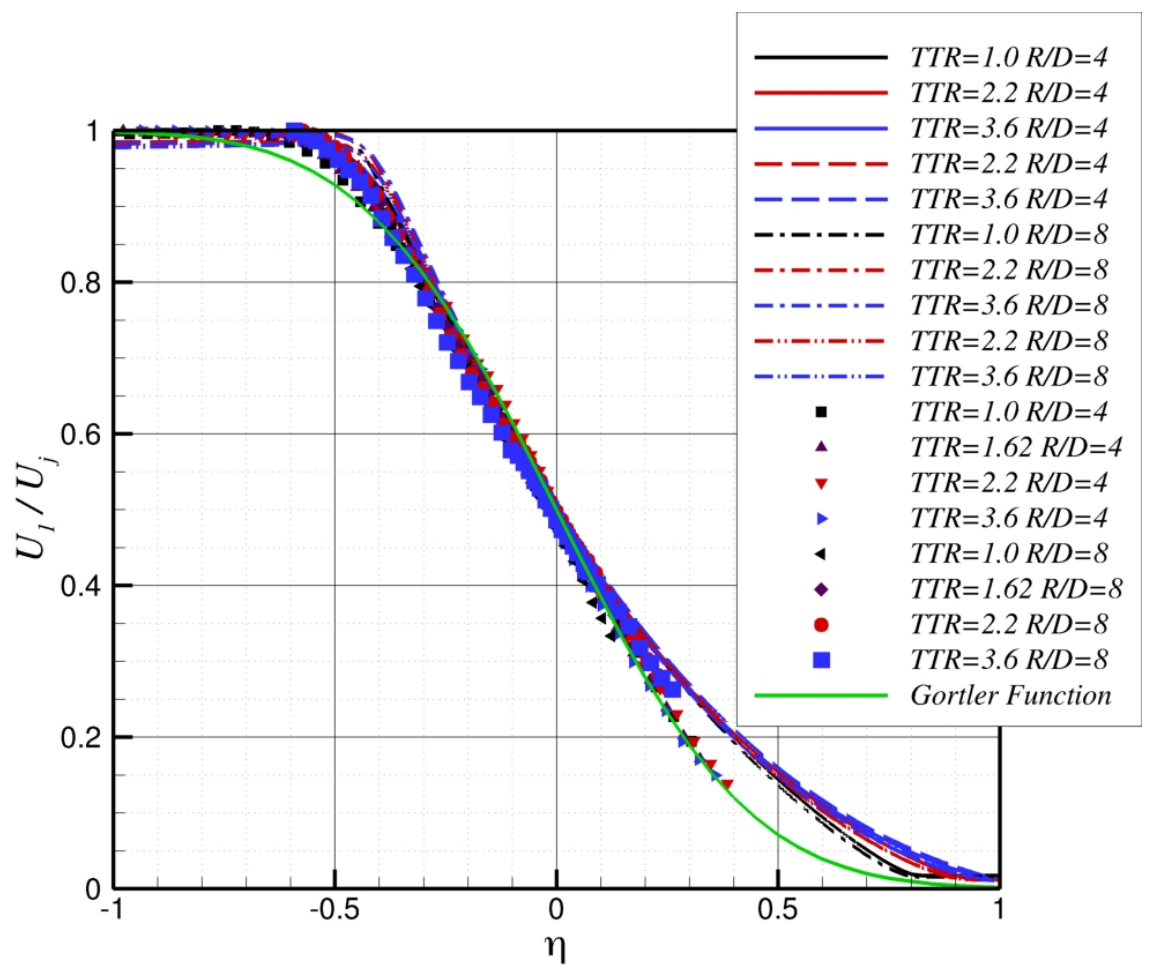

Figure 6. Non-dimensionalized radial mean velocity profiles measured in fully expanded $M_{j}=1.5$ jets of different simulated temperature ratios. 


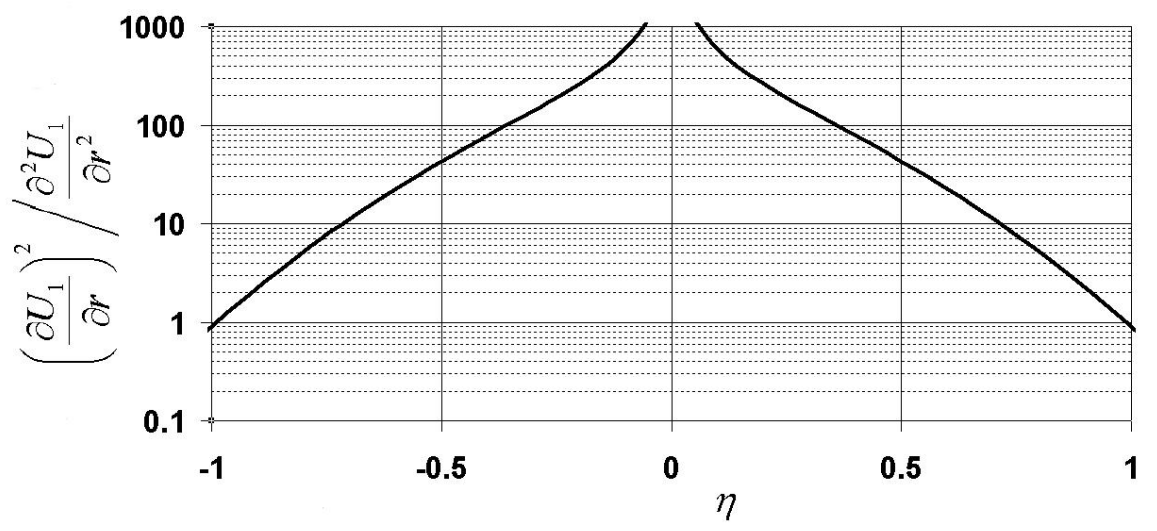

Figure 7. Ratio of the square of the radial derivative of the velocity with its second derivative, as a function of $\eta$

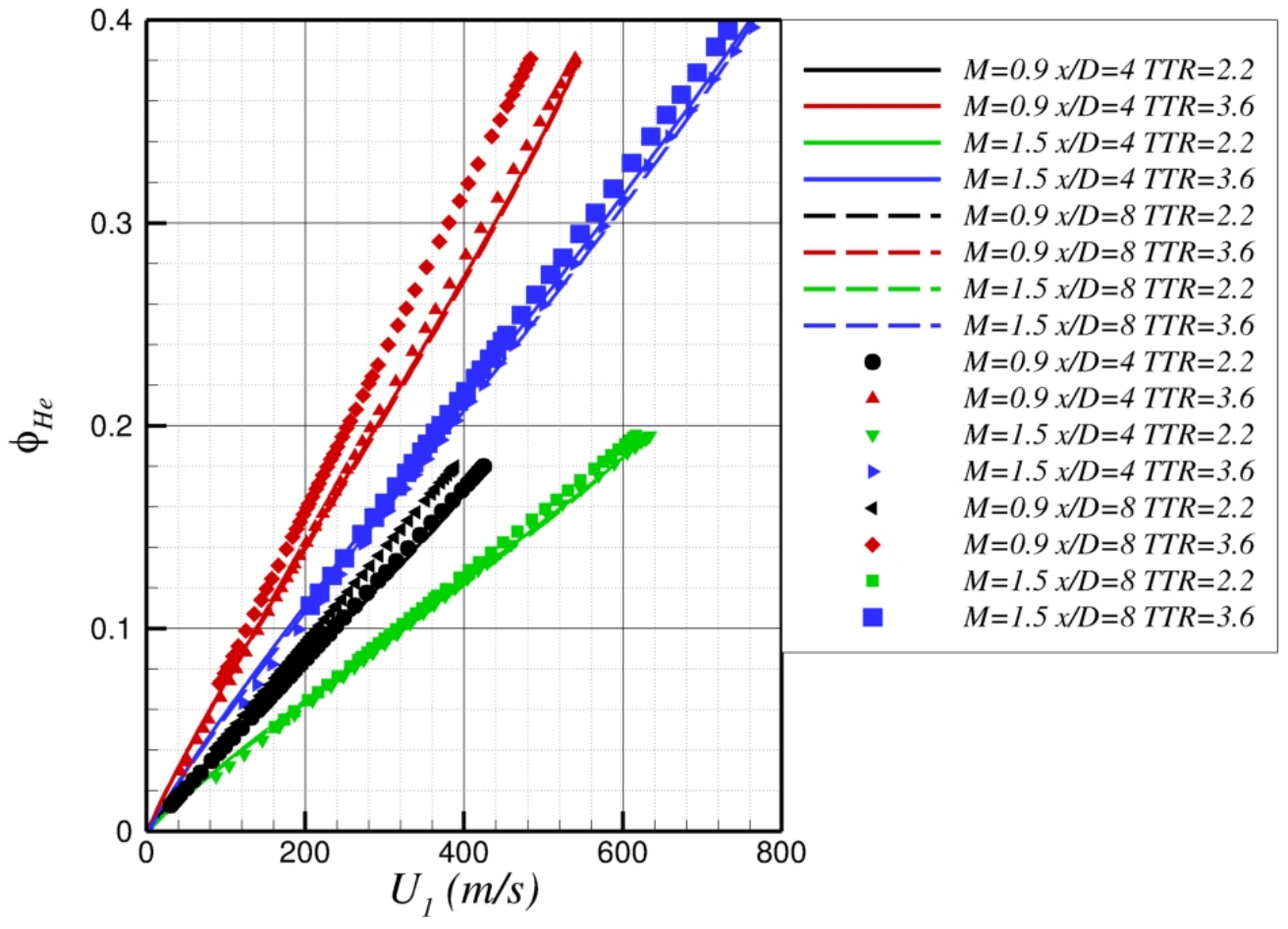

Figure 8. Comparisons of the simulated and experimental helium mass concentration at $x / D=4$ and $x / D=8$ as a function of $u \mathrm{~m} / \mathrm{s}$. Heated simulations, helium-air simulations, and experiments are represented by solid lines, dashed lines, and symbols respectively. 
The helium concentration can also be plotted as a function of the radial position, as shown in Fig. 9 for the experimental conditions of Table 1 and the corresponding helium-air mixture simulations. The values of the helium species are shown at the two downstream measurement locations of $x / D=4$ and $x / D=8$. Good overall agreement between the predictions and experiment can be seen on the centerline and at approximately $r / D=0.5$ for all the jet conditions examined. Unfortunately, as mentioned before, the Menter SST model does not correctly predict the jet spreading rate, resulting in some discrepancies very similar to the ones observed in Figs. 3 and 4. These discrepancies are not due to the additional use of the frozen chemistry model for helium-air mixtures. Unfortunately, the steepened meanflow profiles of the predictions relative to the experimental results has an effect on the helium mass concentration.
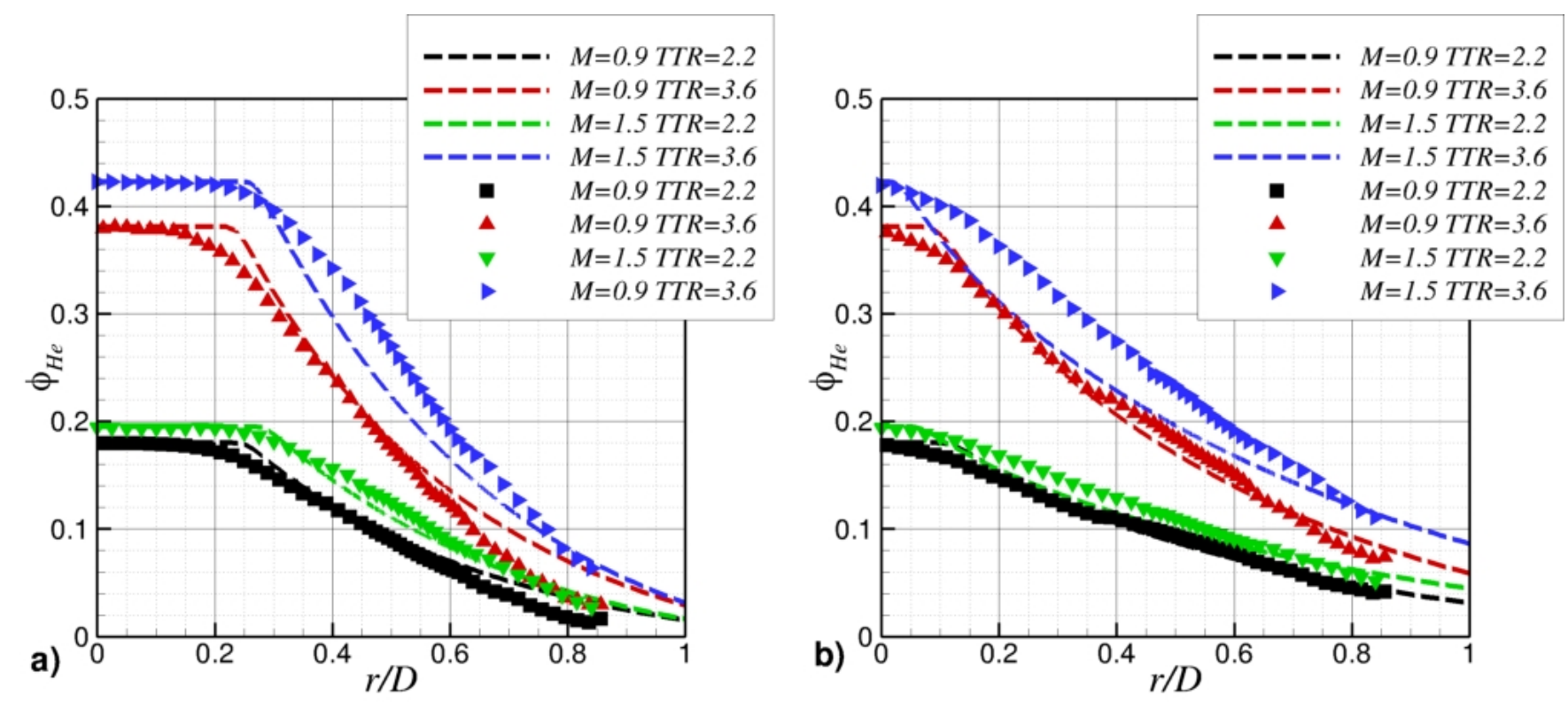

Figure 9. Comparisons of the simulated and experimental helium mass concentration as a function of $r / D$. a) $x / D=4$ b) $x / D=8$. Helium-air simulations and experiments are represented by dashed lines and symbols respectively.

Cross-stream temperature profiles of the $M_{j}=0.9$ jets can be seen in Fig. 10. The solid lines correspond to the static temperature, as obtained from heated air numerical simulations. The experimental points correspond to the static temperature as simulated by the local helium concentration. Similar to the profiles of $U_{1}$, the spatial variables have been normalized by the nozzle diameter $D$. Dimensional units of Kelvin have not been normalized in order to illustrate the differences in simulated temperatures of the various $M_{j}=0.9$ jets. Only the cross-stream profiles at $x / D=4$ are shown, since the simulations become less reliable further downstream. The agreement is excellent at all TTR. Since the simulations were performed for a heated air jet, this constitutes a very good validation of the processing approach used for the experimental data as well as the ability to properly simulate heat with the addition of helium.

If the temperature profiles are normalized by $T_{j}$ and plotted against $\eta$ then self-similarity can be shown for the temperature like the velocity. This is shown in Fig. 11 for the $M_{j}=0.9$ jet at $T T R=2.2$ and $T T R=3.6$. At both $T T R$ the collapse of the numerical predictions and experimental data is very good. Note that $\eta$ is based on $\delta_{w}$ and not the maximum temperature gradient in the cross-stream direction. This fact causes the temperature profiles to not have values of 0.5 at $\eta=0.0$ like the velocity plots. 


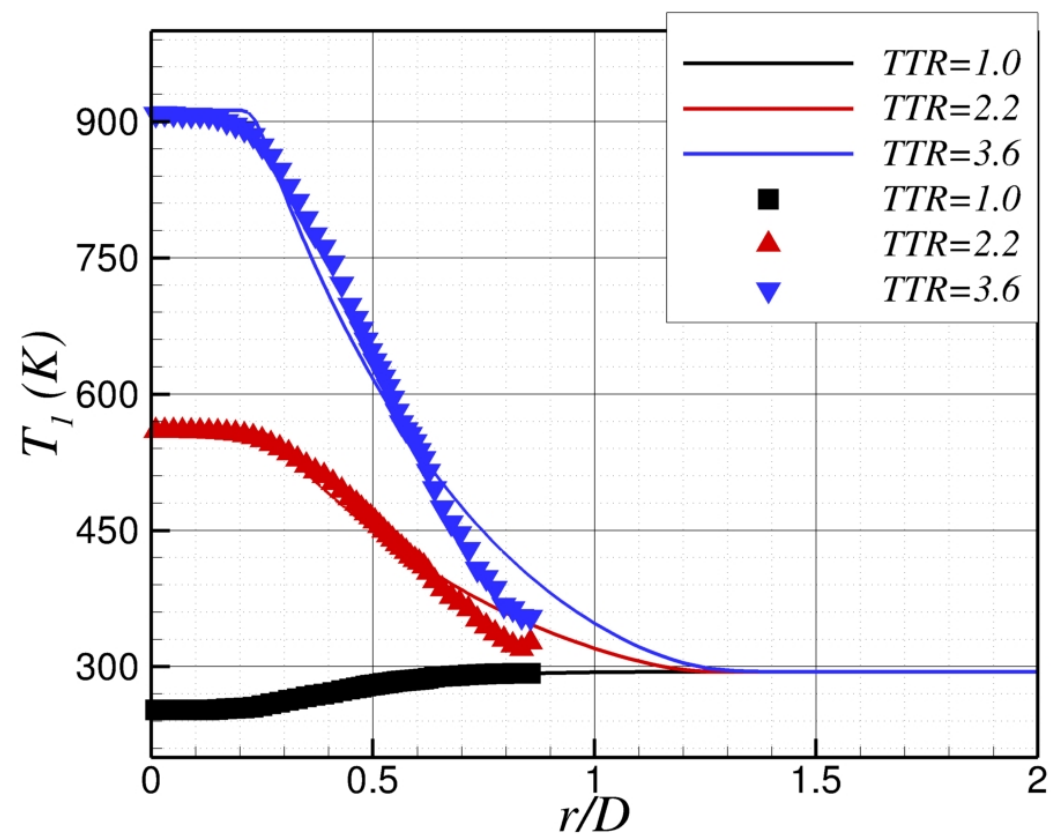

Figure 10. Temperature profiles measured in the $M_{j}=0.9$ jets at $x / D=4$. Heated simulations and experiments are represented by solid lines and symbols respectively.

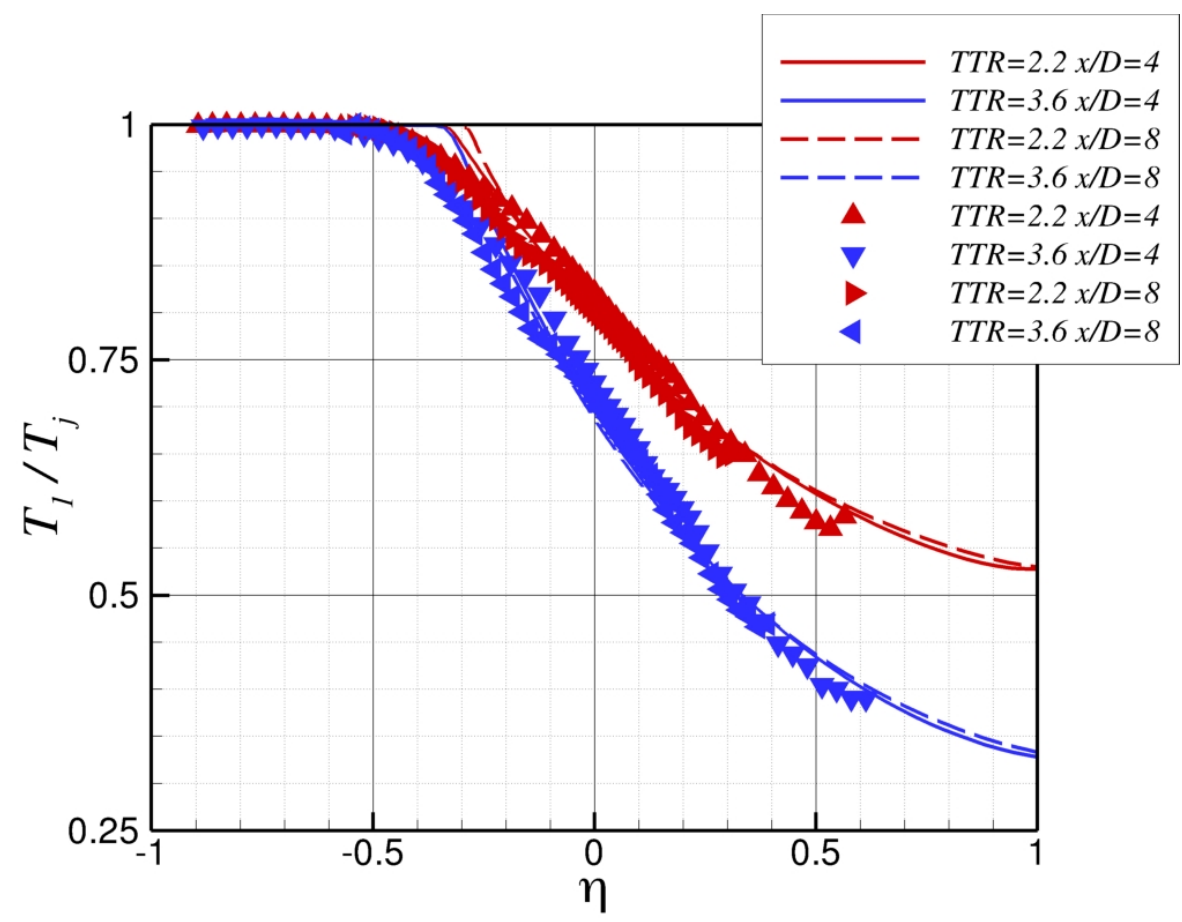

Figure 11. Non-dimensionalized radial mean temperature profiles measured in the fully expanded $M_{j}=0.9$ jet of different simulated temperature ratios. The lines are simulated heated air and the symbols are experimental data. 
Since the local speed of sound is calculated at each radial location of the experimental data, local Mach number $\left(M_{1}\right)$ profiles can be compared with corresponding numerical simulations, as shown in Fig. 12. Figure 12 shows the radial distribution of $M_{1}$ at $x / D=4$ in part a) and $x / D=8$ in part $\mathrm{b}$ ). Both the simulated heated air and helium-air mixture results are also shown. As seen in the cross-stream plots of $U_{1}$, the comparison between the numerical and experimental results shows slight over prediction of $M_{1}$ on the inner and outer parts of the mixing layer, similar to the result for the local velocity $U_{1}$. Slight overprediction of $M_{1}$ on the inner and outer parts of the mixing layer are apparent, as it is for the local velocity $U_{1}$. Similarly, the jet centerline Mach number is obviously over-predicted at $x / D=8$. This is all consistent with the fact that the Menter SST model under-estimates the growth of the mixing layer. The excellent agreement between helium-air and heated air simulations again brings confidence in the accuracy of the frozen chemistry model of Wind-US and the ability to properly simulate the flow of a heated jet with the addition of helium.
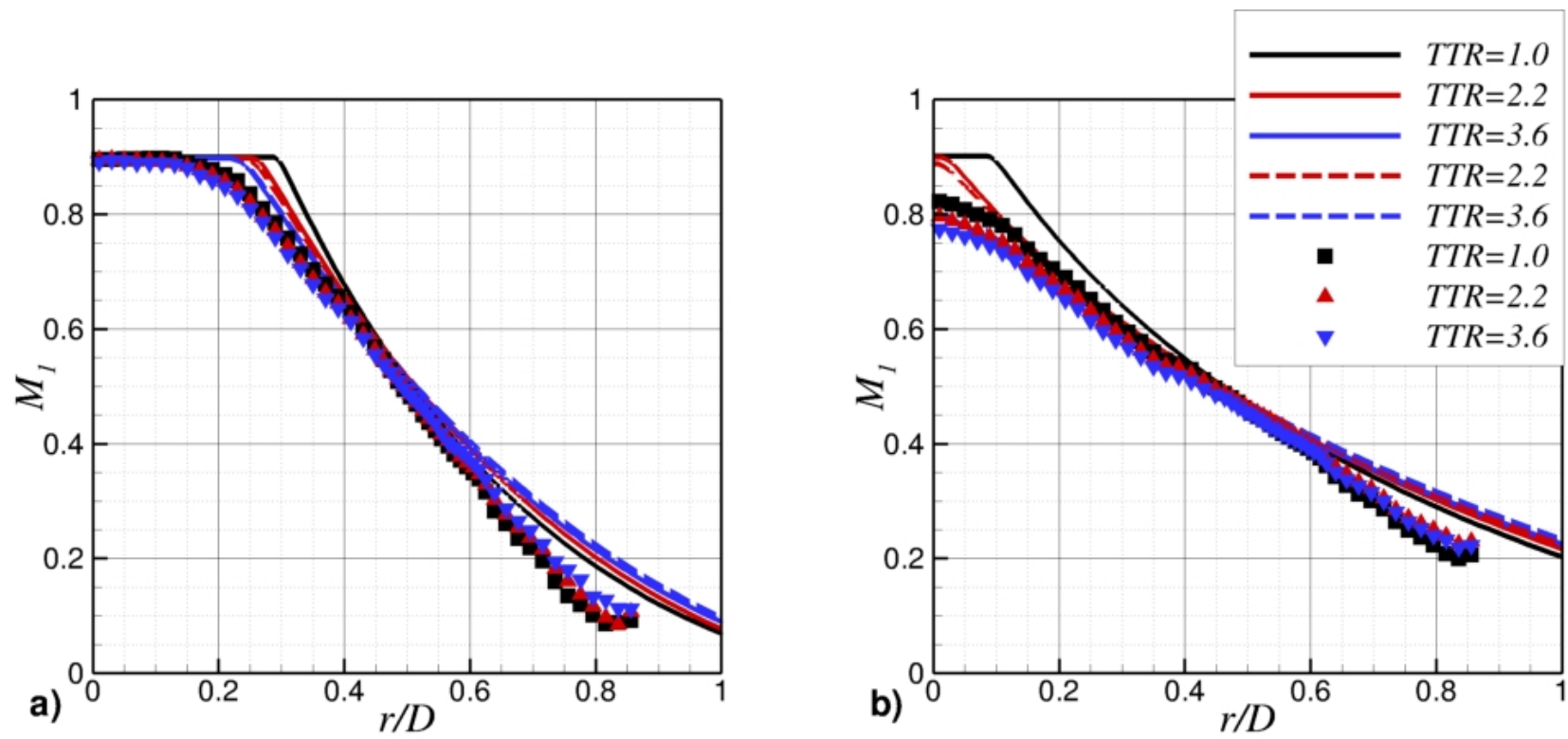

Figure 12. Mach number profiles of the numerical and experimental data for the $M=0.90$ jet operating at various temperature ratios. a) Radial location $x / D=4$. b) Radial location $x / D=8$. Heated simulations, helium-air simulations, and experiments are represented by solid lines, dashed lines, and symbols respectively.

Schlieren images of the $M_{j}=1.5$ jet operating on- and off-design are obtained using helium-air mixtures to simulate the various TTR. Corresponding images from numerical prediction can be constructed by post processing the density flow-field. Constructing these numerical Schlieren requires the derivative of $\rho$ in the cross-stream direction to be calculated. Since the simulations are axisymmetric the result is mirrored across the $x$-axis. In a cartesian $x-y$ coordinate system, because the values of $d \rho / d y$ are essentially opposite across the $x$-axis for an axisymmetric jet, the values of $d \rho / d y$ are multiplied by negative one in the lower half plane. This creates the same light and dark gradients as observed from the Schlieren measurements. The contours of the numerical results are continuous and range from constant values of -6 to 6 , though the values of the density gradients are much larger. These values are selected to better match the colors of the experimental plots. Numerical Schlieren obtained show very good overall agreement with the experimental axisymmetric jets. If the jet is not axisymmetric then three-dimensional flow features may not be apparent in the numerical Schlieren due to the fact that only a computational plane of data may be utilized to create the image (while the experimental Schlieren are an integrated representation of the whole jet).

A comparison of the supersonic $M_{d}=1.0, M_{j}=1.5, T T R=2.2$ jet is shown in Fig. 13(a). The top image in a) is produced experimentally with a Z-type Schlieren setup described in Veltin and McLaughlin. ${ }^{21}$ The images presented result from a large number of spark Schlieren images averaged in order to obtain a better visualization of the shock cell structure and spreading angle. As a side effect, small scale structures are less apparent. Averaging is not required to produce numerical Schlieren. The middle and bottom image of part a) is representative of the numerical Schlieren. The middle numerical Schlieren uses heated air 
and the bottom image shows the helium-air jet for a simulated $T T R=2.2$. Qualitative comparisons may be made by examining the images. In general the on-design case shows good overall agreement with the experiment. The shock cell structure may be faintly seen in the experimental Schlieren but are much more apparent in the numerical simulation. Wind-US inherently assumes that the boundary layer is turbulent and never laminar and no-tripping of the boundary layer occurred in the nozzle interior. This is obviously not representative of the physics of these small jets with Reynolds number around 400,000 that probably possess laminar boundary layers within the entire nozzle length. Since turbulent boundary layers have a larger displacement thickness than laminar ones, the simulation produces slight shocks when operating at $M_{j}=1.5$. The convergent-divergent nozzle used in the experiment is designed to operate with a fully expanded jet and a laminar boundary layer at $M_{d}=1.5$. The shock cell length also exhibits a slight change when operating with a helium-air mixture. Both jets are very close to $M_{j}$ but are slightly different due to very small variations in the flow properties (such as a slightly decreased viscosity due to a different gas species in the nozzle). The off-design parameter of the shock-cell structure is extremely small.

A second Schlieren comparison is made in Fig. 13(b) and illustrates the heated off-design case when $M_{d}=1.0, M_{j}=1.47$, and $T T R=2.2$. The arrangement of the images is the same as part a). This case uses the convergent conical nozzle in both the Schlieren experiment and simulations. The same method to produce the numerical Schlieren of Fig. 13(a) is also used here with the same continuous contours and range. Excellent qualitative agreement may be observed with regard to the shock cell structure position between the predictions and experimental results.

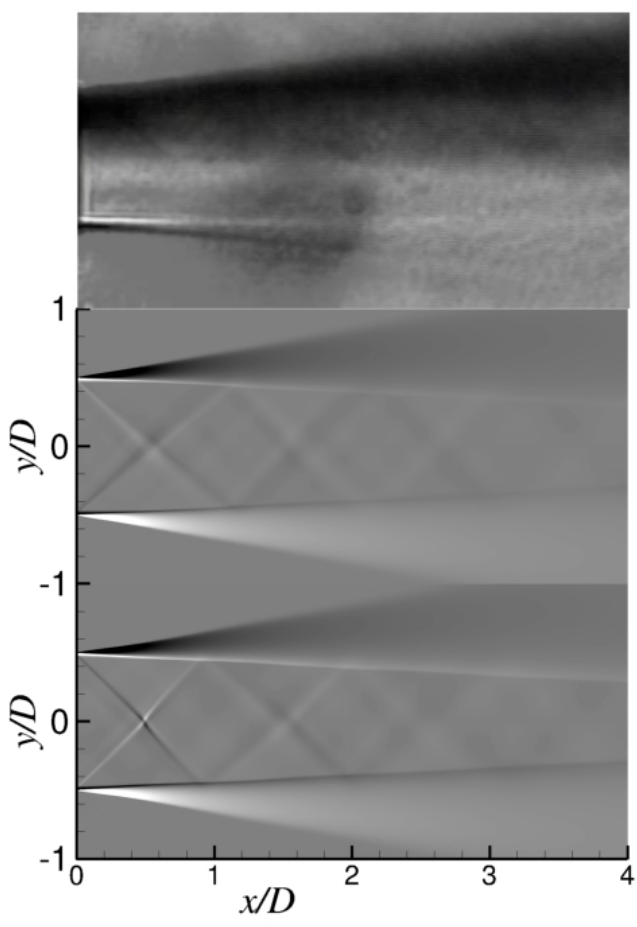

(a) $M_{d}=1.50, M_{j}=1.50, T T R=2.20$ jet.

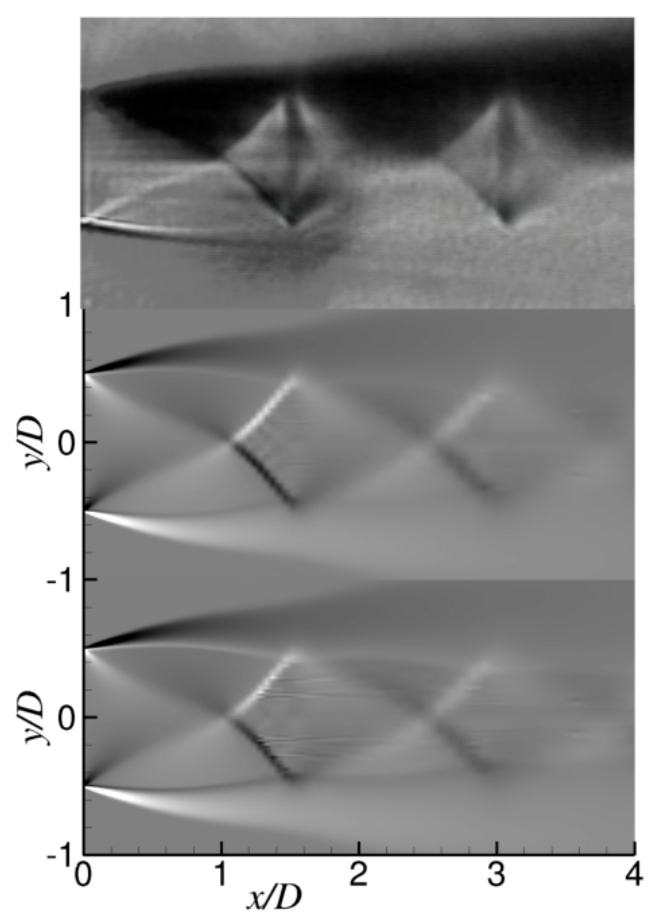

(b) $M_{d}=1.00, M_{j}=1.50, T T R=2.20$ jet.

Figure 13. Comparisons of Z-type Schlieren with numerically generated Schlieren. The top images are produced by the Z-type experimental Schlieren and the middle and bottom are numerically generated results produced by heated air or helium-air mixtures. 
Mach number contour plots are created from the numerical results of the $M_{j}=0.9$ and $M_{j}=1.5$ heated jets at $T T R=2.2$ and $T T R=3.6$. These are illustrated in Figs. 14 through 17. In each figure the top half plane is representative of a simulated heated air result and the bottom half plane is the helium-air result. For the $M_{j}=0.9, T T R=2.2$, and $T T R=3.6$ jet the qualitative agreement between the two jets is excellent. The core lengths and shear layer growth of both the heated air and helium-air mixture are almost the same. The same good agreement of core length and shear layer growth may also be seen in the supersonic cases of Figs. 16 and 17, however, there is some discrepancy between the shock cell structures similar to the observation made from the Schlieren images. These once again, can be attributed to thicker boundary layers and changes in $\gamma$ due to helium in contrast to air.

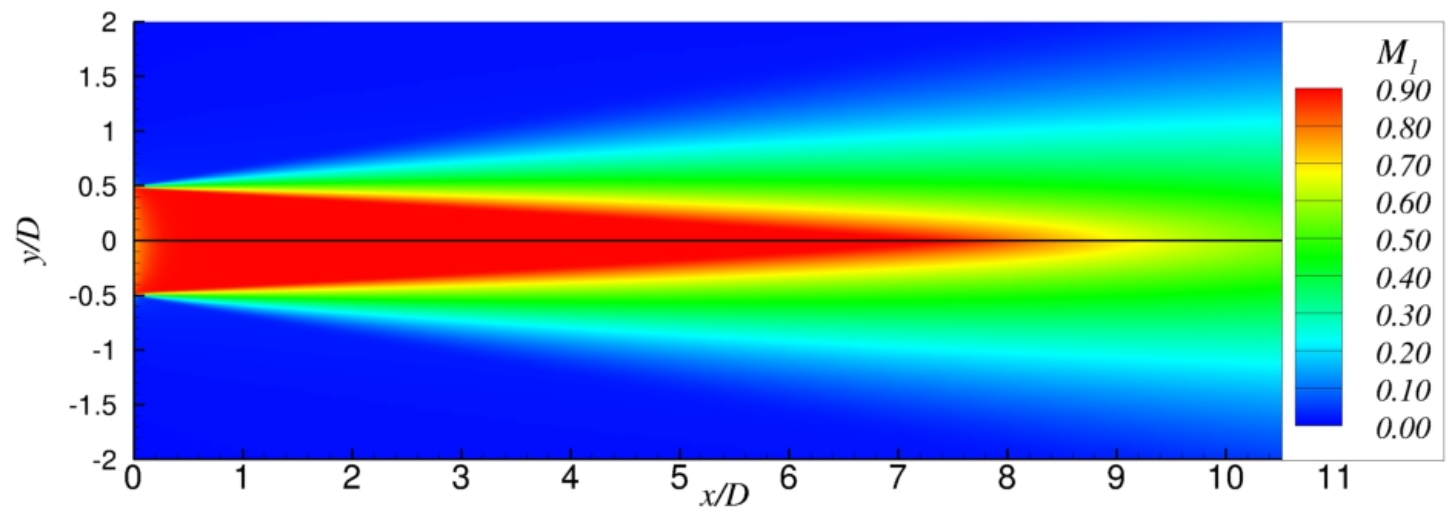

Figure 14. Contour plot of $M$ for the $M_{d}=1.0, M_{j}=0.90, T T R=2.20$ jet. The top half plane, $y>0$ are contours of the heated simulated air jet while the bottom half plane, $y<0$ is representative of contours of the helium-air jet.

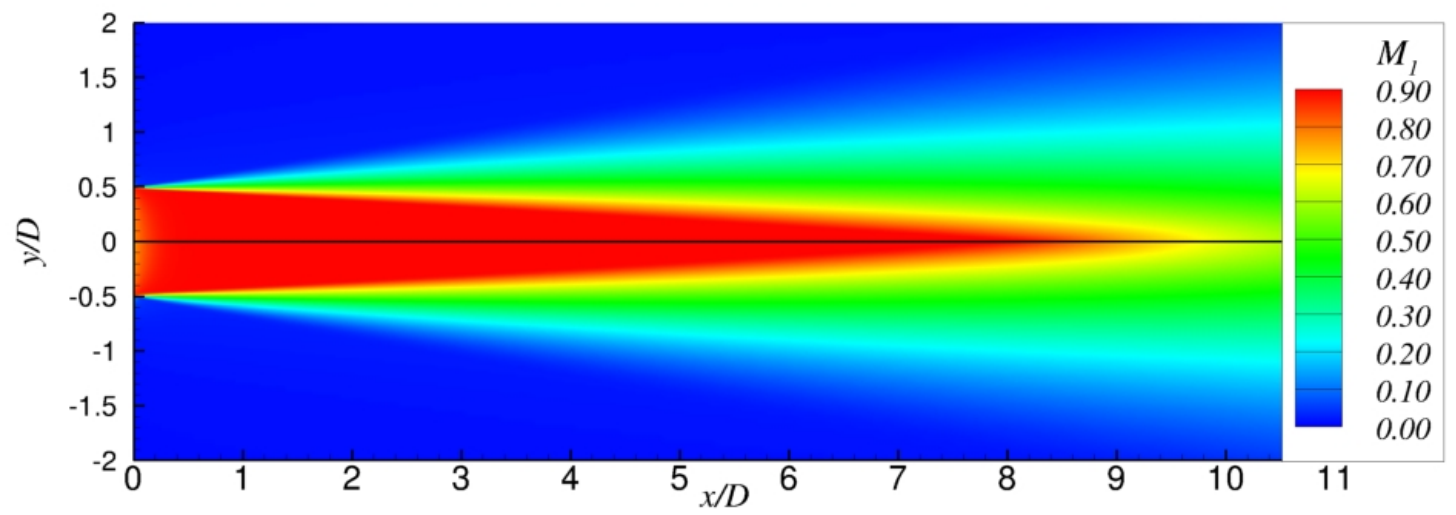

Figure 15. Contour plot of $M$ for the $M_{d}=1.0, M_{j}=0.90, T T R=3.60$ jet. The top half plane, $y>0$ are contours of the heated simulated air jet while the bottom half plane, $y<0$ is representative of contours of the helium-air jet. 


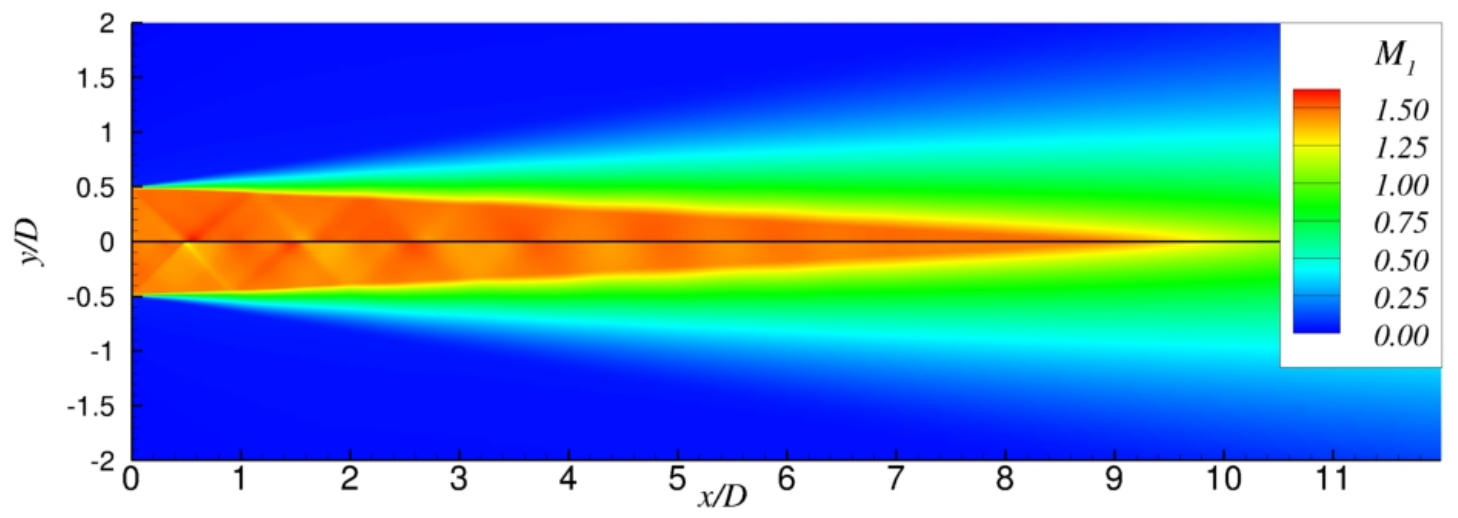

Figure 16. Contour plot of $M$ for the $M_{d}=1.50, M_{j}=1.50, T T R=2.20$ jet. The top half plane, $y>0$ are contours of the heated simulated air jet while the bottom half plane, $y<0$ is representative of contours of the helium-air jet.

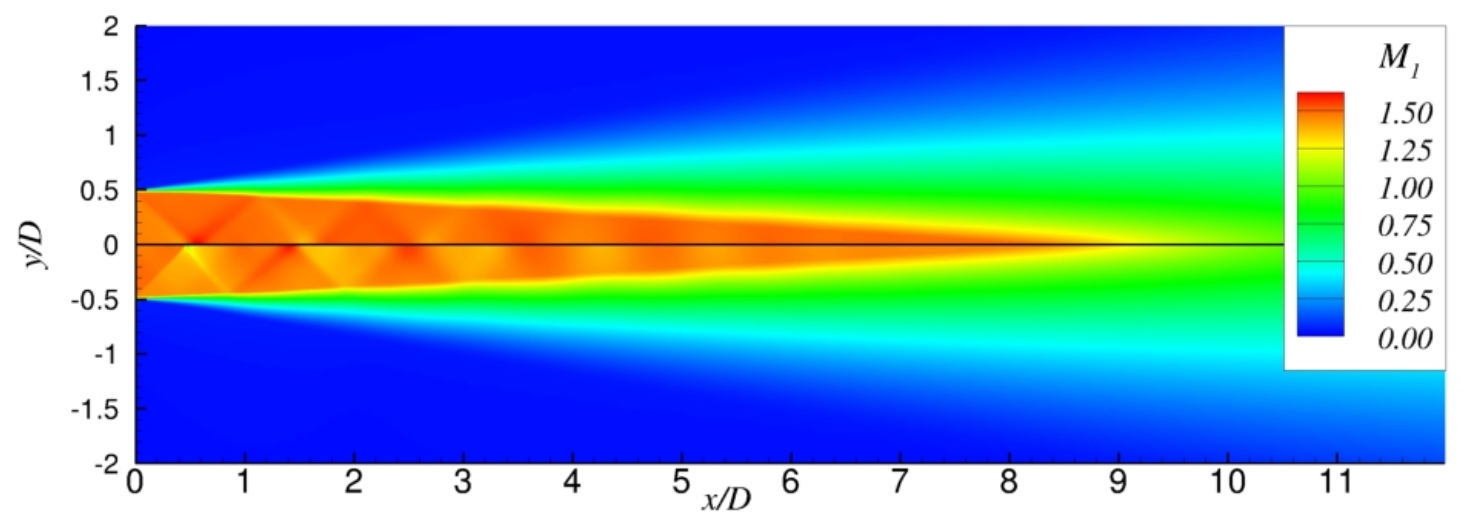

Figure 17. Contour plot of $M$ for the $M_{d}=1.50, M_{j}=1.50, T T R=3.60$ jet. The top half plane, $y>0$ are contours of the heated simulated air jet while the bottom half plane, $y<0$ is representative of contours of the helium-air jet. 
Centerline plots are produced for $\rho_{1}, U_{1}$, and $M_{1}$ from simulations of $M_{j}=0.9$ and $M_{j}=1.5$ heated and heat-simulated jets at $T T R=2.2$ and $T T R=3.6$ in Figs. 18 and 19 respectively. In both figures the rows of sub-figures are representative of $\rho_{1}, U_{1}$, and $M_{1}$ while the columns correspond to the different operating conditions. Since the helium-air concentrations are chosen to match the exit acoustic Mach number $M_{a}$ for both $T T R$ values, the values of $M_{1}$ at the exit of the nozzle $(x / D=0)$ matches perfectly between the mixture and the heated air simulations. For example, in part g) of Fig. $18 M_{1}$ is almost identical along the centerline axis. The same excellent agreement can be seen in other cases also. Only for the supersonic cases does the core extend slightly farther with the helium-air jets compared to the heated air jets. As expected, comparisons of the density for both temperature ratios and three corresponding jet conditions do not exhibit the same level of agreement. This is due to the fact previously stated that it is not possible to match both the density and the Mach number. While these simulations are performed by matching $M_{a}$ between heated air and helium-air jets, the calculation could easily have been performed by matching the exit densities. Nevertheless, for each TTR and NPR the discrepancy in the predicted values of $\rho_{1}$ remains very small.

The values of the streamwise velocity, $U_{1}$, on the centerline also agree very favorably, as seen in Figs. 18 and 19 for both $T T R$ and $M_{j}$ values. These plots in particular illustrate the very slight changes in core length of the off-design calculation. Still, the core length remains almost exactly the same between the heated air and helium-air mixture for the subsonic calculations and supersonic on-design calculations.

The centerline values of the supersonic on-design jet of heated air and helium-air demonstrate that the core lengths are exactly the same. Also, it can be seen in part h) of both figures that the values of $M_{1}$ at the jet exit are almost exactly the same value. Even with both of these observations, for the supersonic on-design jet, the helium-air jet is slightly over-expanded while the heated air simulated jet is under-expanded. The rise or fall of velocity due to shocks or Prandtl-Meyer expansion waves can be seen in the centerline plots. These values of $M_{j}$ at the jet exit are extremely close to the design Mach number $M_{d}=1.5$. Their slight difference produces shock cells of different lengths, as already observed from the Schlieren images and the Mach number contour plots. When examining the discontinuities of $M_{1}$ in part h) of both Figs. 18 and 19, it appears that there is an initial very strong shock. However, this is an artifact of using the axisymmetric boundary condition where $\partial P_{1} / \partial r=0$. This condition is theoretically true at a point; however, it is enforced in CFD by using grid points that are on the centerline axis and the corresponding grid points of the spatial discretization stencil in the cross-stream direction. Therefore, there is a numerical effect of broadening the region where $\partial P_{1} / \partial r=0$. This effect can be varied by changing the grid spacing in the cross-stream direction near the centerline, effectively causing a barrel shock that has the diameter on the order of twice the distance from the centerline to the first grid point in the cross-stream direction. To minimize this effect, the grid point clustering in the cross-stream direction on the centerline axis uses the same distribution as the nozzle wall at $x / D=0$. 

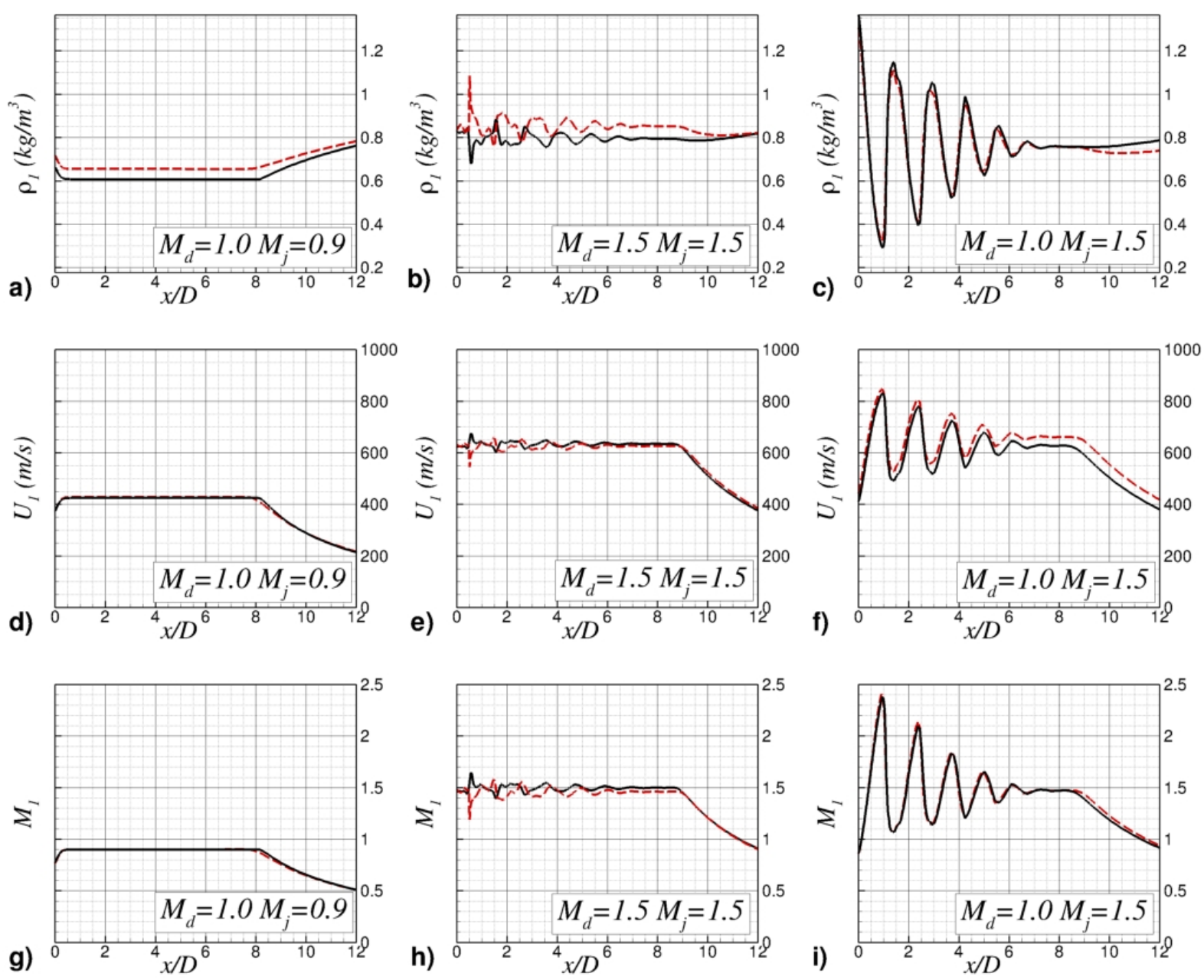

Figure 18. Various centerline comparisons of $T T R=2.2$ jets. - air $-—$ helium-air. 

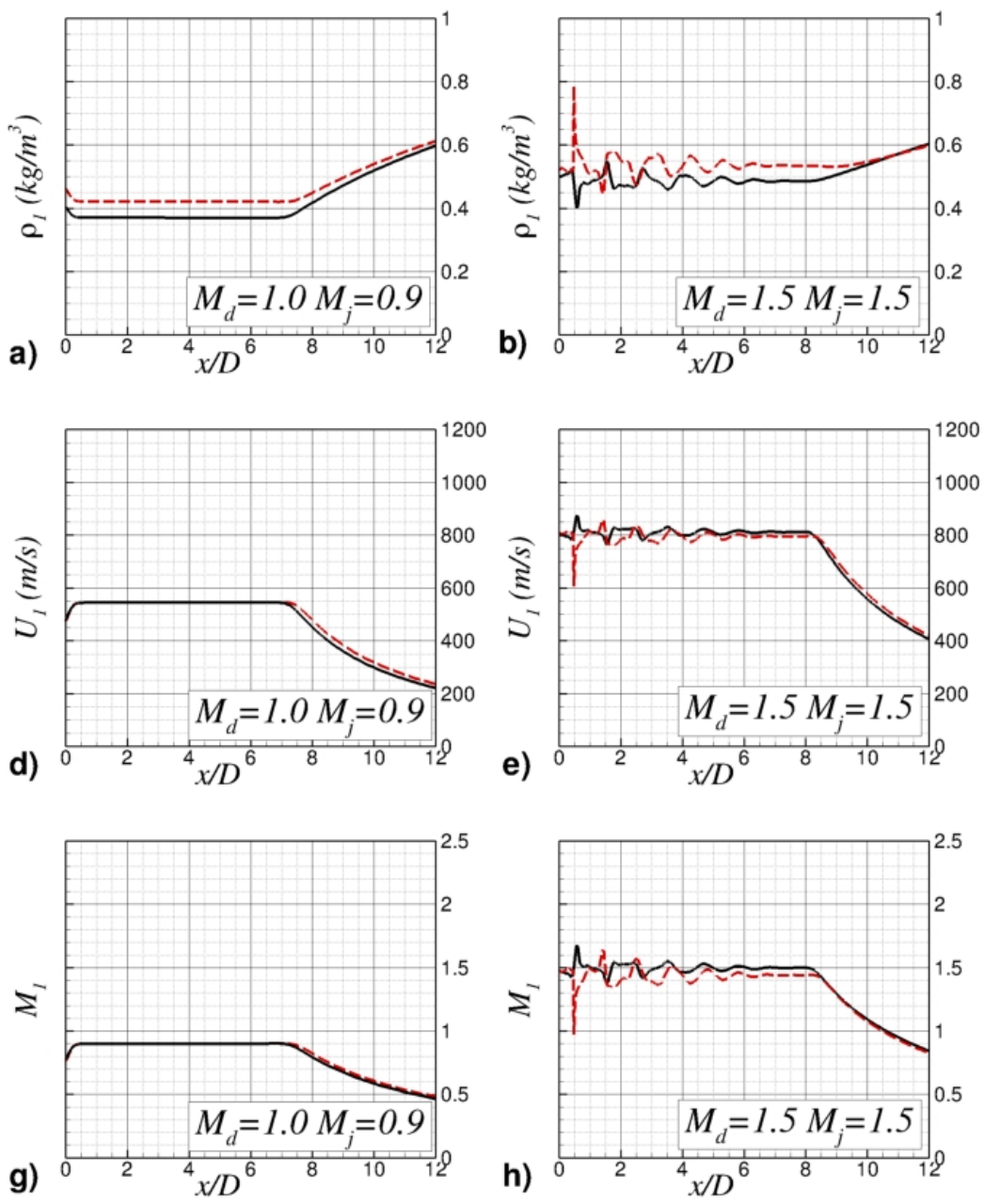
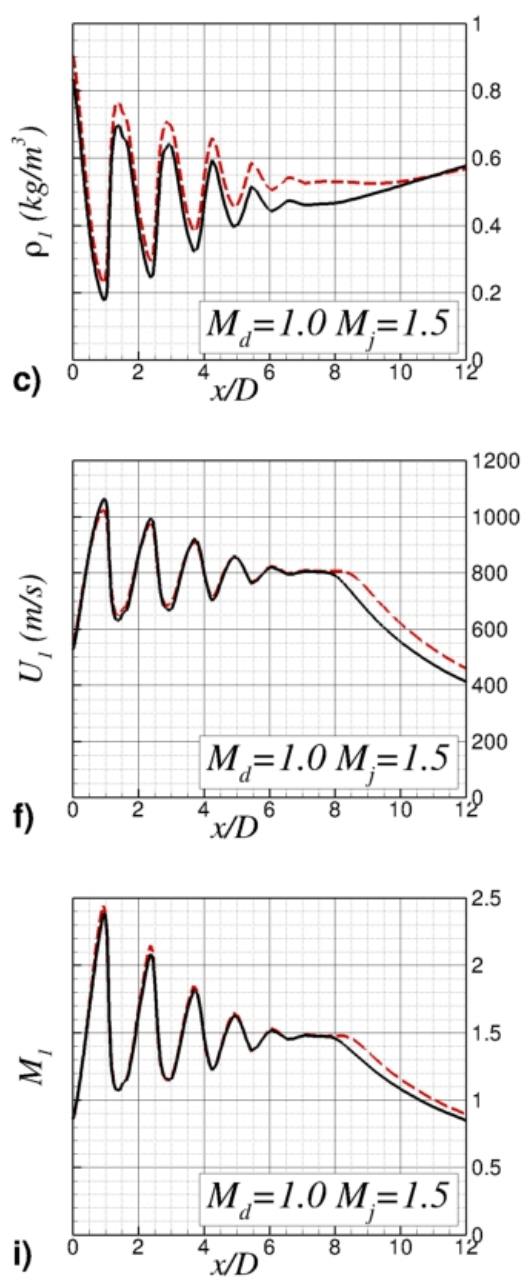

Figure 19. Various centerline comparisons of $T T R=3.6$ jets. - air -- helium-air. 


\section{Conclusion}

Experimental and numerical assessment of the flow properties of high speed helium-air mixture and heated air jets were presented. High subsonic and supersonic shock-free and shock-containing jets were considered and demonstrated a very good agreement between the numerical simulations and experiments. The flow experimentally assessed in helium-air jets has properties very similar to heated air jets measurements present in the literature. The turbulent model used for the simulation was observed to underpredict the growth rate of the jet in particular for the lower jet Mach number cases, leading to a mis-prediction of the potential core length. However, the very good agreement between simulated heated air and helium-air mixture jets provide a validation of the frozen chemistry model used, as well as further evidence that the helium addition does indeed provide mean flow properties similar to heated air.

\section{Acknowledgments}

The experiments were partially supported through the NRA Cooperative Agreement NNX07AC88A. The Technical Monitor for the Cooperative Agreement is Dr. Milo Dahl of the NASA Glenn Research Center. The authors would like to express their gratitude to Prof. Philip Morris and Prof. Dennis McLaughlin for their consultation as well as Nick Georgiadis of the NASA Glenn Research Center for helpful discussions regarding the Wind-US code. Joo Kyung Suh of Raytheon Missile Systems is acknowledged for assembling the chemistry coefficient file.

\section{References}

${ }^{1}$ Tanna, H. K., Dean, P. D., and Fisher, M. J., "The Influence of Temperature on Shock-Free Supersonic Jet Noise," Journal of Sound and Vibration, Vol. 39, No. 4, 1975, pp. 429-460.

${ }^{2}$ Lau, J., "Effects of Exit Mach Number and Temperature on Mean-Flow and Turbulence Characteristics in Round Jets," Journal of Fluid Mechanics, Vol. 105, 1981, pp. 193-218.

${ }^{3}$ Seiner, J. M., Ponton, M. K., Jansen, B. J., and Lagen, N. T, "The Effects of Temperature on Supersonic Jet Noise Emission," German Society for Aeronautics and Astronautics (DGLR)/AIAA Paper 92-02-046, 1992.

${ }^{4}$ Tam, C. K. W., and Chen, P., "Turbulent Mixing Noise from Supersonic Jets," AIAA Journal, Vol. 32, No. 9, 1994, pp. 1774-1780.

${ }^{5}$ Doty, M. J., and McLaughlin, D. K, "Acoustic and Mean Flow Measurements of High Speed Helium Air Mixture Jets," International Journal of Aeroacoustics, Vol 2, No.3, 2003, pp293-334.

${ }^{6}$ Kinzie, K., McLaughlin, D.K., "Measurement of Supersonic Helium-Air Mixture Jets," AIAA Journal, Vol. 37, No. 11, 1999, ,pp. 1363-1369.

7 Papamoschou, D, "Acoustic Simulation of Coaxial Hot Air Jets Using Cold Helium-Air Mixture Jets," Journal of Propulsion and Power, Vol. 23, No. 2, March-April 2007, pp. 375-381.

${ }^{8}$ Bridges, J., Brown, C. A., "Validation of the Small Hot Jet Acoustic Rig for Aeroacoustic Research," $11^{\text {th }}$ AIAA/CEAS Aeroacoustics Conference, AIAA Paper 2005-2846, 2005.

${ }^{9}$ Kuo, C.-W., Veltin, J., and McLaughlin, D. K., "Acoustic Measurements of Models of Military Style Supersonic Nozzle Jets," $4^{\text {th }}$ AIAA Aerospace Sciences Meeting Including The New Horizons Forum and Aerospace Exposition, AIAA-2009-18, 2009.

${ }^{10}$ Miller S.A.E., Veltin J., Morris P.J., McLaughlin D.K., "Assessment of Computational Fluid Dynamics for Supersonic Shock Containing Jets," AIAA Journal, Vol. 47, No.11, pp. 2738-2746.

${ }^{11}$ Spalding, D.B., "Concentration fluctuations in a round turbulent free jet", Chemical Engineering Science, Vol. 26, 1971, pp. $95-107$.

${ }^{12}$ Schlichting, H. and Gersten, K., "Boundary layer theory," Springer, 2003.

${ }^{13}$ Panchapakesan, N.R. and Lumley, J.L., "Turbulence Measurements in Axisymmetric Jets of Air and Helium. Part 2. Helium Jet," Journal of Fluid Mechanics, Vol. 246, 1993, pp. 225-247.

${ }^{14}$ Yimer, I., Campbell, I., Jiang, L.-Y., "Estimation of the Turbulent Schmidt Number from Experimental Profiles of Axial Velocity and Concentration for High Reynolds Number Jet Flows," Canadian Aeronautics and Space Journal, Vol. 48, No. 3, 2002.

${ }^{15}$ Lau, J., Morris, P.J., Fisher. M.J., "Measurements in Subsonic and Supersonic Free Jets using a Laser Velocimeter," Journal of Fluid Mechanics, Vol. 93, 1979, pp. 1-27 pp. 193-218.

${ }^{16}$ Menter, F. R., "Two-Equation Eddy-Viscosity Turbulence Models for Engineering Applications," AIAA Journal Vo. 32. No. 8, Aug. 1994, pp. 1598-1605.

${ }^{17}$ Yoder, D. A., Georgiadis, N. J., and O'Gara, M. R. "Frozen Chemistry Effects on Nozzle Performance Simulations," $38^{t h}$ Fluid Dynamics Conference and Exhibit, 23-26 June, Seattle, Washington, AIAA 2008-3909, 2008.

${ }^{18}$ The NPARC Alliance, NASA Glenn Research Center, Cleveland, Ohio, "Wind-US Users Guide," 2009.

${ }^{19}$ McBride, B. J., Gordon, S., and Reno, M. A., "Thermodynamics Data for Fifty Reference Elements," NASA TM-3287, January, 1993.

${ }^{20}$ Nelson, C. and Power, G., "The NPARC Alliance Flow Simulation System," AIAA Paper 2001-0594, January 2001.

${ }^{21}$ Veltin, J. and McLaughlin, D. K., "Noise Mechanisms Investigation in Shock Containing Screeching Jets Using Optical Deflectometry," 14th AIAA/CEAS Aeroacoustics Conference, AIAA Paper 2008-2889, Vancouver, Canada, May, 2008. 